\title{
Qualitative Results on the Dynamics of a Berger Plate with Nonlinear Boundary Damping
}

\author{
Pelin G. Geredeli * Justin T. Webster ${ }^{\dagger}$
}

February 1, 2016

\begin{abstract}
The dynamics of a (nonlinear) Berger plate in the absence of rotational inertia are considered with inhomogenous boundary conditions. In our analysis, we consider boundary damping in two scenarios: (i) free plate boundary conditions, or (ii) hinged-type boundary conditions. In either situation, the nonlinearity gives rise to complicating boundary terms. In the case of free boundary conditions we show that well-posedness of finite-energy solutions can be obtained via highly nonlinear boundary dissipation. Additionally, we show the existence of a compact global attractor for the dynamics in the presence of hinged-type boundary dissipation (assuming a geometric condition on the entire boundary [23]). To obtain the existence of the attractor we explicitly construct the absorbing set for the dynamics by employing energy methods that: (i) exploit the structure of the Berger nonlinearity, and (ii) utilize sharp trace results for the Euler-Bernoulli plate in [25].

We provide a parallel commentary (from a mathematical point of view) to the discussion of modeling with Berger versus von Karman nonlinearities: to wit, we describe the derivation of each nonlinear dynamics and a discussion of the validity of the Berger approximation. We believe this discussion to be of broad value across engineering and applied mathematics communities.
\end{abstract}

Keywords: Global attractors, nonlinear plate equation, well-posedness, asymptotic behavior of dynamical systems

AMS Mathematics Subject Classification 2010: 35Q74, 74K20, 35B41, 35A01

\section{Introduction}

We consider the well-posedness and long-time behavior of solutions to a boundary damped Berger plate equation taken in the absence of rotational inertia. Its derivation and relation to the scalar von Karman nonlinearity [14, 15, 23] are discussed in depth in Section 1.1. We consider the isotropic plate to be thin, as is usual in large deflection theory [23. Thus we consider a two dimensional domain

\footnotetext{
*Hacettepe University, Dept. of Mathematics, 06800 Beytepe, Ankara, Turkey; pguven@hacettepe.edu.tr

${ }^{\dagger}$ College of Charleston, Dept. of Mathematics, Charleston, SC; websterj@cofc.edu
} 
$\Omega \subset \mathbb{R}^{2}$ with smooth boundary $\Gamma$. The partial differential equation (PDE) model of interest is then:

$$
\begin{cases}u_{t t}+\Delta^{2} u+\left[\gamma-\Upsilon \cdot\left(\int_{\Omega} \nabla u \cdot \nabla u d \Omega\right)\right] \Delta u=p(\mathbf{x}) & \text { in }(0, T) \times \Omega \\ B C(u) & \text { on } \Gamma \\ u(0)=u_{0} ; \quad u_{t}(0)=u_{1} . & \end{cases}
$$

The term $p \in L^{2}(\Omega)$ represents static pressure on the top surface of the plate. The physical parameter $\Upsilon$ is fixed and positive, while $\gamma$ corresponds to in-plane stretching $(\gamma<0)$ or compression $(\gamma>0)$. In this treatment (i) we consider the case $\gamma \geq 0$, as is by now standard in treatments of the Berger plate [10, 13, and (ii) we normalize $\Upsilon=1$. The term $B C(u)$ in (1.1) represents the plate's boundary conditions.

Throughout the paper, we will consider nonlinear damping acting through two distinct types of boundary conditions; the nature of damping will be critical to our well-posedness and long-time behavior results.

- Free-clamped boundary conditions with dissipation, denoted (FCD):

$$
\left\{\begin{array}{l}
\Delta u+(1-\mu) B_{1} u=0 \text { on } \Gamma_{1}, \\
\partial_{\nu}(\Delta u)+(1-\mu) B_{2} u-\mu_{1} u=\left|u_{t}\right|^{6} u_{t} \text { on } \Gamma_{1}, \\
u=\partial_{\nu} u=0 \text { on } \Gamma_{0},
\end{array}\right.
$$

where $\Gamma=\Gamma_{0} \sqcup \Gamma_{1}$ is a disjoint union, $\Gamma_{0} \neq \emptyset$, and the boundary operators $B_{1}$ and $B_{2}$ are given by [14, 23]:

$$
\begin{aligned}
& B_{1} u=2 \nu_{1} \nu_{2} u_{x y}-\nu_{1}^{2} u_{y y}-\nu_{2}^{2} u_{x x}=-\partial_{\tau \tau} u-(\operatorname{div} \nu) \partial_{\nu} u, \\
& B_{2} u=\partial_{\tau}\left[\left(\nu_{1}^{2}-\nu_{2}^{2}\right) u_{x y}+\nu_{1} \nu_{2}\left(u_{y y}-u_{x x}\right)\right]=\partial_{\tau} \partial_{\nu} \partial_{\tau} u .
\end{aligned}
$$

$\nu=\left(\nu_{1}, \nu_{2}\right)$ is the unit outer normal to $\Gamma, \tau=\left(-\nu_{2}, \nu_{1}\right)$ is the choice of unit tangent vector along $\Gamma$. The parameter $\mu_{1}$ is nonnegative ${ }^{1}$. The constant $0<\mu<1$ has the meaning of the Poisson modulus.

- Hinged boundary conditions with dissipation, denoted (HD):

$$
u=0 ; \quad \Delta u=-D\left(\partial_{\nu} u_{t}\right) \text { on } \Gamma,
$$

where the damping function $D(\cdot) \in C^{1}(\mathbb{R})$ is monotone increasing, and $D(0)=0$.

Remark 1.1. It is clear that, barring issues of elliptic regularity, one can consider various combinations of the above boundary conditions. (This is done, for instance, throughout [14.) In the analysis of (FCD), we consider a disjoint portion of the boundary to be clamped, primarily to simplify aspects of our analysis which are not the principle focus, and to avoid technical issues of elliptic regularity. One can take the entire boundary to be free, so long as $\mu_{1}>0$.

Remark 1.2. Another configuration (which is of physical interest) involves the so-called hinged-clamped boundary conditions. One can consider the damping acting via moments on a portion of the boundary

\footnotetext{
${ }^{1}$ Since $\Gamma_{0} \neq \emptyset$, we could take $\mu_{1}=0 \underline{14}$, however, we retain it for the sake of generality.
} 
$\Gamma_{1}$ (again, disjoint from $\Gamma_{0}$-where the boundary is clamped). This configuration, denoted by (HCD), is given by:

$$
\begin{cases}u=0 ; & \partial_{\nu} u=0 \text { on } \Gamma_{0} \\ u=0 ; & \Delta u=-D\left(\partial_{\nu} u_{t}\right) \text { on } \Gamma_{1}\end{cases}
$$

We remark further on the (HCD) configuration in Remark 1.8 below.

For the Berger and von Karman evolutions, taken with homogeneous boundary conditions (of clamped or hinged-type), the theory is rather complete. However, in the two inhomogeneous cases considered here the results we obtain differ dramatically from those available for other nonlinear plate equations (e.g., von Karman, Kirchhoff); this occurs since the advanced theory for these evolution equations does not apply. In fact, the inhomogeneous boundary conditions are what differentiate present analysis of the Berger plate from those available in other available literature (see Section 1.2).

We seek to determine the effect of boundary damping on the dynamics, and seek asymptotic behavior results (when possible) under "standard" assumptions on the plate domain and damping functions. We also provide a discussion of the validity of the Berger model under these boundary contributions, including a review of the pertinent engineering and mathematical literature. By providing a modern analysis of the Berger evolution with dissipation acting on the boundary, we attempt to reconcile the hurdles in PDE analysis with those described in the engineering literature. Indeed, the engineering literature reviewed here provides numerical contrast between the von Karman and Berger models in the presence of each of the standard boundary conditions.

Notation: We will make use of standard Sobolev spaces $W^{s, p}(\Omega)$ and denote norms in $H^{s}(\Omega)(p=2)$ as $\|\cdot\|_{s}$ and $\|\cdot\|_{0}=\|\cdot\|_{L^{2}(\Omega)}$. We will use the notation $(\cdot, \cdot)_{\Omega}$ for inner-products in $L^{2}(\Omega)$ and $\langle\cdot, \cdot\rangle_{\Gamma}$ for those in $L^{2}(\Gamma)$ (or a subset of $\Gamma$, as indicated by the subscript where necessary). For simplicity, norms and inner products written without subscript are taken to be $L^{2}$ of the appropriate domain (e.g., $(\cdot, \cdot)$ on $\Omega$ and $\langle\cdot, \cdot\rangle$ on $\Gamma$ ). The space $W_{0}^{s, p}(\Omega)$ denotes the completion of $C_{0}^{\infty}(\Omega)$ in the $W^{s, p}(\Omega)$ norm. We will use the ubiquitous constant $C>0$, and denote critical dependencies when necessary.

\subsection{Modeling Considerations}

This portion of the paper is expository. It discusses the origins of the von Karman and Berger nonlinearities, and provides an engineering perspective on the validity of each model.

Let $u^{i}(x, y, z, t)$ denote the $i$ th component of the displacement vector of the middle surface of an elastic plate occupying the region $\Omega \subset \mathbb{R}^{2}$. Let $\epsilon_{i j}$ and $\sigma_{i j}$ denote the standard strain and stress tensors, respectively ${ }^{2}$. We assume the plate is homogeneous and isotropic (with associated stress-strain relation).

The so called full von Karman system is based on the employment of a variation principle applied to the energy, and the assumption of finite elasticity [23, Ch.2, pp. 13-20]. This is a modification of the Kirchhoff approach, which is itself based on (i) a linear strain-displacement relation and (ii) an assumption that the linear filaments of the plate remain perpendicular to the central plane of the plate throughout deflection. (The second hypothesis is then linearized to produce the Kirchhoff equation.) The full von Karman system is arrived at by replacing (i) above with the nonlinear strain-displacement

\footnotetext{
${ }^{2}$ Here, and below, we utilize the summation convention and identify the spatial variables $x=x_{1}, y=x_{2}$, and $z=x_{3}$.
} 
relation

$$
\epsilon_{i j}=\frac{1}{2}\left(\frac{\partial u^{i}}{\partial x_{j}}+\frac{\partial u^{j}}{\partial x_{i}}\right)+\frac{1}{2} \frac{\partial u^{k}}{\partial x_{i}} \frac{\partial u^{k}}{\partial x_{j}} .
$$

The resulting system (which has a nice vectorial representation - see [22] and references therein) consists of a nonlinear beam equation in the transverse displacement, coupled (nonlinearly) to a system of elasticity for the in-plane displacements; much work has been done on this system [22, 29] (and references therein). To simplify the structure of these equations, one may neglect the in-plane acceleration components of the model [14, 15. This allows for the decoupling of the in-plane and transverse dynamics. The resulting nonlinearity is referred to as the scalar von Karman nonlinearity:

$$
f_{V}(u)=-\left[u, v(u)+F_{0}\right]
$$

where $F_{0}$ is a given in-plane load. The von Karman bracket is employed:

$$
[u, v]=u_{x x} v_{y y}+u_{y y} v_{x x}-2 u_{x y} v_{x y}
$$

for all functions $u, v$ sufficiently smooth [14]. The Airy stress function, $v(u)$ solves

$$
\Delta^{2} v=-[u, u] \text { in } \Omega ; v=\partial_{\nu} v=0 \text { on } \Gamma \text {. }
$$

Remark 1.3. We note that in the studies of nonlinear plates often rotational inertia in the filaments of the plate (represented by the term $-\left(h^{2} / 12\right) \Delta u_{t t}$ on the LHS of the plate equation (1.1), where $h$ is the thickness of the plate) is neglected. When $h$ is small (the plate is thin), this term is discardable [15, 23. Mathematically, however, this term is highly non-trivial and has substantial bearing on well-posedness and long-time behavior results.

Even with the simplification to a scalar equation, the complex structure of the scalar von Karman nonlinearity (making use of a nonlinear elliptic solver) provides incentive to attempt further simplification. We now paraphrase a review in [28]:

In 1955, Berger proposed in [6] a modified von Karman system. This simplification is based upon the assumption that the so called second strain invariant of the middle surface is negligible. The resulting equations are simpler than von Karman's and Berger compared his results to known solutions, and found good agreement. This approach was generally accepted, though the hypothesis lacked a clear mechanical interpretation. Yet, in many problems solved via the Berger method the edges of the plates were assumed to be restrained from in-plane movements, and, numerically, the exactness of the method is associated to such restrictions. For freely movable edges the accuracy of the results furnished by the Berger approach becomes questionable. Indeed, the resulting [numerical] accuracy depends on the order of the differential operators appearing in the boundary conditions. In this way, hinged and free boundary conditions provide more opportunities [than clamped conditions] for the solutions to become inaccurate or singular.

Another analysis in [32] attempts to find a rational mechanical basis for the Berger method. However, the authors also conclude that the Berger results may not be a consistently valid approximations across types of boundary conditions. In [5], it is elaborated that Berger's line of thought leads to questionable results for movable edge conditions owing to the fact that the neglect of second strain 
invariant with movable edges fails to imply freedom of rotation in the middle plane where the elastic stress exists. The novelty of [5] is that the authors proceed to provide an additional nonlinear term in the equations of motion to correct this, which they report has good accuracy with known solutions for all types of boundary conditions.

\subsection{Previous Results and Mathematical Motivation}

We now provide a general overview of previous mathematical work done on the system in 1.1. Note that none of the results below focus on inhomogenous boundary conditions; to our knowledge no previous works have provided a PDE analysis of the Berger plate with dissipation acting on the boundary - though such analyses are prevalent for the more "complex" von Karman evolution.

The classical work in [16] establishes results on the existence of exponential attractors for a more general Berger-like equation in $\mathbb{R}^{n}$ (which subsumes the standard Berger model), taken with homogeneous hinged boundary conditions and fully-supported interior damping. This reference discusses early work done by Ball (see references in [16]) on extensible beam equations. The methods utilized by the authors of [16] rely on the development of modified energy functionals which allow one to circumvent the need for an explicit construction of a Lyapunov function 3

In the more recent book [11, the Berger evolution with homogeneous hinged boundary conditions (along with more general nonlinear terms) is considered with fully-supported interior, linear damping. Utilizing a Lyapunov approach, the existence of smooth attractors is shown, but only in the presence of (regularizing) rotational inertia terms.

In [7] the authors study a non-dissipative von Karman model arising in the context of piston theory [8, 14 for nonlinear flow-plate interactions. This work also considers the case where rotational inertia is absent, and the primary difficulties arise via (i) free boundary conditions, as well as (ii) non-dissipative (piston theory) terms appearing (1.1). The analysis does not concern the Berger evolution, yet the authors must also combat non-dissipative terms in the setting of free boundary conditions.

In [26, a Berger beam is considered with free-clamped boundary conditions; in this work stabilization is accomplished via a nonlinear boundary feedback acting in the third order free boundary condition. We also point out that to accommodate the non-dissipative terms which arise due to the interaction of the geometric nonlinearity and the free boundary condition (as discussed herein), the author incorporates the nonlinear boundary contribution into the boundary condition at the free end of the beam. Whether this is physical is unclear, however it does present an interesting mathematical consideration which could be investigated for free-clamped Berger plates.

The analysis in [19] deals with the boundary stabilization of a Kirchhoff (polynomial) nonlinear plate, taken with rotational inertia, and linear damping via a hinged boundary condition (analogous to what we take). Though the analysis is somewhat simplified (due to rotational inertia), this work provides a guide for handling the non-dissipative, second-order trace terms in the case of hinged boundary conditions below.

Finally, in perhaps the most modern account, the expansive monograph [14 addresses the wellposedness and long-time behavior of the scalar von Karman equations. However, this text also contains preliminary material on linear plates, and an abstract presentation of nonlinear plates. Owing to this,

\footnotetext{
${ }^{3}$ This is a key issue below: in many nonlinear plate/extensible beam problems the construction of a (strict) Lyapunov function requires a "unique continuation" result, which may not be generally available [17, 18.
} 
many of the results on well-posedness and long-time behavior are valid for Berger's evolution in the case of clamped or hinged type boundary conditions. However, if any type of free boundary condition is employed (homogenous or inhomogeneous), the analysis in [14, although vast, does not apply. Moreover, the best results in [14] for the von Karman evolution (with no rotational inertia terms) with hinged dissipation (HD) concerns local compact attractors. (In this reference they actually address the more general (HCD) conditions - allowing for a clamped portion and hinged dissipation portion (Remark 1.2 - with a geometric condition in force on $\Gamma_{0}$; their results apply to (HD) with no geometric condition by simply taking $\Gamma_{0}=\emptyset$.) To obtain attractors in a global sense, additional assumptions must be made (fully-supported interior damping), and the resulting approach is indirect. For a more detailed discussion relating these results to those here, see the discussion following the presentation of Theorem 1.3 below.

In this treatment we are analyzing two cases where the Berger dynamics have a marked difference from the von Karman dynamics. In the case of free-clamped (FCD) type boundary conditions, Berger's nonlinearity produces an additional non-dissipative term in the dynamics which can only be accommodated via a highly nonlinear damping to obtain well-posedness of the model. As such, Section 3 can be viewed as a quantifiable mathematical manifestation of the difficulties described above in applying Berger's approximation to the von Karman model in the presence of a free boundary component. For our analysis, we assume Berger's hypothesis is in force and we analyze 1.1) directly from the equations, yielding non-dissipative effects which enter the analysis at the level of finite energy.

In the case of hinged-type boundary conditions (HD), after showing the well-posedness of the model (similar to what is done for (FCD)), we are interested in the asymptotic-in-time analysis. The standard nonlinear dissipation acting via moments on the boundary is sufficient to obtain ultimate compactness of the dynamics (in the form of a global attractor) without any additional damping or non-standard assumptions - though we do require a somewhat classical geometric assumption on the domain (albeit for a new reason: the interaction between (HD) and the nonlinearity). In fact, we explicitly construct the absorbing ball using techniques from [12, and along with modern techniques in the theory of dynamical systems (related to the asymptotic smoothness property [14, 21], see Sec. 4.2), we obtain the existence of a compact global attractor.

\subsection{Functional Setting}

The natural energy for linear plate dynamics is given by

$$
E(t)=E\left(u, u_{t}\right)=\frac{1}{2}\left\{\left\|u_{t}(t)\right\|^{2}+a(u(t), u(t))\right\}
$$

Here, $a(u, v)$ represents the potential energy and given by the bilinear form

$$
a(u, v)=\widetilde{a}(u, v)+\mu_{1} \int_{\Gamma_{1}} u v,
$$

where

$$
\widetilde{a}(u, v) \equiv \int_{\Omega}(\Delta u \Delta v-(1-\mu)[u, v])
$$


Again, the notation $[u, v]$ corresponds to the von Karman bracket (as introduced earlier in the text), and for all functions sufficiently smooth [14]:

$$
\int_{\Omega}[u, v] d \Omega=-\int_{\Gamma}\left(\left(B_{1} u\right) \partial_{\nu} v-\left(B_{2} u\right) v\right) d \Gamma .
$$

Typically $\mu<1 / 2$, but we allow for $\mu \leq 1$ and note that $\mu=1$ corresponds to the limiting case for the boundary conditions, and the bilinear form $\widetilde{a}(\cdot, \cdot)$ collapses to the standard bilinear form $(\Delta \cdot, \Delta \cdot)_{\Omega}$ in the case of (HD) boundary conditions.

The above energy $E(t)$ dictates the state space

$$
\mathscr{H} \equiv \begin{cases}H_{\Gamma_{0}}^{2}(\Omega) \times L^{2}(\Omega) & \text { for }(F C D), \\ \left(H^{2} \cap H_{0}^{1}\right)(\Omega) \times L^{2}(\Omega) & \text { for }(H D) .\end{cases}
$$

Throughout this treatment, we will use the following nonlinear energies associated to (1.1):

$$
\mathscr{E}(t)=\mathscr{E}\left(u, u_{t}\right)=E(t)+\Pi(u(t)), \quad \widehat{E}(t)=E(t)+\frac{1}{4}\|\nabla u(t)\|^{4},
$$

where $\Pi$ represents the non-dissipative and nonlinear portion of the energy

$$
\Pi(u)=\frac{1}{4}\left(\|\nabla u\|^{4}-2 \gamma\|\nabla u\|^{2}-4 \int_{\Omega} p u\right) .
$$

\subsection{Main Results and Discussion}

In this treatment the main results concern: well-posedness under (FCD) and (HD) boundary conditions, as well as long time behavior of the dynamics corresponding to 1.1 under (HD) boundary conditions.

Firstly, consider equation (1.1) with (FCD). We now give a novel well-posedness result (see Section 2 for formal definitions of solutions):

Theorem 1.1. With reference to (1.1), taken with (FCD) boundary conditions and with initial data $\left(u_{0}, u_{1}\right) \in \mathscr{H}$, for any $T>0$ there exists a unique generalized solution $u \in C(0, T ; \mathscr{H})$ satisfying the energy equality

$$
\mathscr{E}(t)+\int_{s}^{t} \int_{\Gamma_{1}}\left|u_{t}\right|^{8}+\int_{s}^{t} \int_{\Gamma_{1}}\left(\gamma-\|\nabla u\|^{2}\right)\left(\partial_{\nu} u\right) u_{t}=\mathscr{E}(s)
$$

for every $t>s>0$. The dynamics depend continuously on the initial data. This implies that the map $\left(u(0), u_{t}(0)\right) \mapsto\left(u(t), u_{t}(t)\right)$ defines a strongly continuous semiflow $S_{F}(t)$ on $\mathscr{H}$.

Remark 1.4. We emphasize that with only linear boundary damping the global existence of the solutions is an open problem, as one cannot obtain the necessary a priori bounds. To overcome this difficulty we consider a nonlinear boundary dissipation which has an extreme (yet minimal with respect to our analysis) polynomial structure.

Secondly, we analyze the Berger model with (HD) boundary conditions. It should be noted that under (HD) conditions (unlike the (FCD) conditions) the structure of equation 1.1) does not yield any 
non-dissipative terms in the energy relation. This makes the direct application of the abstract plate theory more straightforward for obtaining a well-posedness result. Before giving this, the following assumption is needed on the damping:

Assumption 1. There exists positive constants $0<m<M<\infty$ such that

$$
m \leq D^{\prime}(s) \leq M, \quad|s| \geq 1
$$

Remark 1.5. For the purposes of well-posedness alone, one could impose weaker assumptions on the damping mechanism $D(s)$; e.g., $D(s)$ could exhibit arbitrary polynomial growth. However, for our subsequent results concerning long time behavior it is necessary that $D(s)$ satisfy the stronger assumption above which is also utilized in [12, 14].

Now we give the well-posedness result of 1.1 taken with (HD) boundary conditions:

Theorem 1.2. Let Assumption 1 hold. With reference to (1.1) taken with (HD) boundary conditions and with initial data $\left(u_{0}, u_{1}\right) \in \mathscr{H}$, for all $T>0$ there exists a unique generalized solution $u \in$ $C(0, T ; \mathscr{H})$ depending continuously on the initial data. This implies that the map $\left(u(0), u_{t}(0)\right) \mapsto$ $\left(u(t), u_{t}(t)\right)$ defines a strongly continuous semiflow $S_{H}(t)$ on $\mathscr{H}$. The following energy equality holds:

$$
\mathscr{E}(t)+\int_{s}^{t} \int_{\Gamma} D\left(\partial_{\nu} u_{t}\right)\left(\partial_{\nu} u_{t}\right)=\mathscr{E}(s)
$$

Remark 1.6. Assumption 1 provides linear bounds for the damping function from below and above which ensures the validity of the energy equality (for more detail, see [14, Section 4.2]).

Existence and properties of compact attractors for nonlinear plate models (e.g., von Karman, Berger, Kirchhoff) have been studied in various contexts over the last 30 years ${ }^{4}$ In the present case, the dynamical system associated to the Berger dynamics with (FCD) cannot easily be shown to be dissipative. The absence of a strict Lyapunov function (not readily available) seems to preclude showing the existence of a compact global attractor for the dynamics. However, we can show dissipativity of the dynamical system under (HD) boundary conditions. Thus, by showing the asymptotic smoothness property of the associated dynamical system, the plate dynamics under (HD) will admit the existence of a compact global attractor for finite energy, generalized solutions. For this, we need an additional, standard, star-shaped geometric assumption on the boundary [14, 23]:

Assumption 2. There exists a point $\mathbf{x}_{0} \in \mathbb{R}^{2}$ such that $h(\mathbf{x})=\mathbf{x}-\mathbf{x}_{0}$ has the property that $h \cdot \nu \geq 0$ for all $\mathbf{x} \in \Gamma$, where $\nu$ is the outward normal vector to $\Gamma$.

Remark 1.7. The star-shaped condition given above arises in boundary control, when the boundary (or just a portion) is subjected to a given feedback. In using state-of-the-art trace estimates [25], we can accommodate boundary terms associated with the linear dynamics without a geometric condition. However, to deal with the nonlinear Berger contribution on the boundary (in utilizing the flux multiplier $h \cdot \nabla u$ ), we utilize the star-shaped assumption on $\Gamma$. Throughout [23] a star-shaped and star-complemented geometric condition (what is in Assumption 2, with the additional assumption that

\footnotetext{
${ }^{4}$ See Section 1.2 also see the Appendix for a discussion of dynamical systems and concepts relevant to the treatment here.
} 
$h(\mathbf{x}) \cdot \nu \leq 0$ on $\left.\Gamma_{0}\right)$ is in force for obtaining uniform decay rates. Such a star-shaped, star-complemented assumption is also used for various configurations in the context of von Karman equations in [14]. We note that our assumption on $\Gamma$ is precisely what [25] can eliminate for linear dynamics while still obtaining the same results of [23]; however, at present it seems indispensable to have an assumption on the boundary $\Gamma$ due to the interaction of the nonlinearity and the boundary conditions.

We note that at present the best result obtained for non-rotational von Karman dynamics in the (HD) configuration (with additional damping and assumptions) is that of local attractors (14, Theorem 10.5.7]). This is to say that when one restricts the semiflow $\left(S_{H}(\cdot), \mathscr{H}\right)$ to a ball of radius $R$ of initial data, a compact attractor can be shown for the restricted dynamical system. This attractor is not necessarily uniform with respect to large $R$. Such a result follows (though not immediately) from the so called asymptotic smoothness property of the dynamics, and estimates which yield a local absorbing ball. (See [14, Section 10.5] for this presentation.) To obtain a global attractor the authors of [14] utilize the fact that a gradient dynamical system, which is also asymptotically smooth, has a compact global attractor (Theorem 6.2 in the Appendix here). To show the gradient property of the dynamics, they rely upon (additional) fully-supported interior damping. This damping yields a "unique continuation" property for the dynamics (or, from another point of view, allows for "uniqueness" in a compactnessuniqueness argument). This approach, and the requisite additional damping, is necessary because "direct multipliers" do not yield a uniform absorbing ball for the von Karman dynamics - owing to boundary contributions and the structure of the $\Pi$ for von Karman dynamics.

In the case of Berger's nonlinearity, we are able to adapt an approach in [12] to show the existence of an absorbing set for the entire dynamics. This is precisely because of the structure of the Berger nonlinearity. The discussion above brings us to the primary result concerning long-time behavior of the Berger dynamics with (HD) boundary conditions:

Theorem 1.3. Consider (1.1) taken with (HD) boundary conditions; under Assumptions 1 and 2 , the dynamical system $\left(S_{H}(t), \mathscr{H}\right)$ has a compact global attractor.

Remark 1.8. The above holds in the case of (HCD) boundary conditions (see Remark 1.2), where $\Gamma=\Gamma_{0} \sqcup \Gamma_{1}$ and the dissipation is active only on $\Gamma_{1}$. This will be addressed in the forthcoming manuscript [1], which will also investigate further properties (such as dimensionality and smoothness) of the attractor given in Theorem 1.3. The key point in analyzing the partially-damped boundary configuration is to address the higher-order trace term $\Delta u$ on the uncontrolled portion of the boundary $\Gamma_{0}$. A geometric condition on the entire boundary (for instance, the star-complemented, star-shaped condition mentioned in Remark 1.7) is not sufficient in obtaining estimates which control the size of the absorbing ball. Rather, precise control of $\left.\Delta u\right|_{\Gamma_{0}}$ must be obtained via techniques which can be found, for instance, in 2, 3] (and references therein).

\subsection{Technical Challenges and Contributions}

With regard to much of the multiplier analysis, Berger's nonlinearity behaves more nicely than von Karman. And, in the case of homogeneous hinged or clamped boundary conditions, the analysis of the von Karman plate essentially subsumes the analysis of the Berger evolution. However, under (FCD) 
boundary conditions (with $F_{0} \in H_{0}^{2}(\Omega)$ ), we have the disparity:

$$
\begin{aligned}
\left(f_{V}(u), u_{t}\right)_{\Omega}=\left(-\left[u, v(u)+F_{0}\right], u_{t}\right)_{\Omega} & =\frac{1}{2} \frac{d}{d t}\left\{\frac{1}{2}\|\Delta v(u)\|_{\Omega}^{2}-\left(\left[u, F_{0}\right], u\right)_{\Omega}\right\} \\
\left(f_{B}(u), u_{t}\right)_{\Omega}=\left(\left(\gamma-\|\nabla u\|_{\Omega}^{2}\right) \Delta u, u_{t}\right)_{\Omega} & =\frac{1}{2} \frac{d}{d t}\left\{\frac{1}{2}\|\nabla u\|_{\Omega}^{4}-\gamma\|\nabla u\|_{\Omega}^{2}\right\} \\
& +\left(\gamma-\|\nabla u\|^{2}\right)\left\langle\partial_{\nu} u, u_{t}\right\rangle_{\Gamma_{1}} .
\end{aligned}
$$

The boxed term highlights the fundamental difference of the dynamics, as the Berger-free dynamics contain an inherently non-dissipative term with which we must contend.

In the case of (HD) boundary conditions, we note that the terms of interest arise from the biharmonic term - which plays a key role in the multiplier analysis. We have for any $\phi$ sufficiently smooth

$$
\left(\Delta^{2} u, \phi\right)_{\Omega}=(\Delta u, \Delta \phi)_{\Omega}+\left\langle\partial_{\nu}(\Delta u), \phi\right\rangle_{\Gamma}+\left\langle D\left(\partial_{\nu} u_{t}\right), \partial_{\nu} \phi\right\rangle_{\Gamma}
$$

In this case of $\phi=h \cdot \nabla u$ (for $h$ a "nice" vector field, $u \in H^{2}(\Omega)$ ) the damping on the boundary is pitted against a high order boundary term, which must be accommodated for any sort of long-time behavior analysis. Such trace terms must be handled delicately. Here, we are able to (via the special structure of the Berger nonlinearity) control these boundary terms directly, in a way that allows techniques from [12] to obtain.

\subsubsection{Principal Challenges}

The non-dissipative term above for (FCD) in 1.12 appears in the energy relation for the dynamics (and it is absent for von Karman dynamics). We follow the modern approach in [14, using the abstract plate theory developed therein, and we must utilize fully nonlinear damping of high polynomial degree. As such, we must address the technical difficulties associated to such damping when utilizing energy methods. For obtaining dissipativity and asymptotic smoothness of the dynamical system associated to the (HD) dynamics, we need to handle the nonlinear damping carefully.

In both configurations - (FCD) and (HD) — obtaining the results in Section 1.4 requires addressing the criticality (with respect to the finite energy topology) of the Berger nonlinearity. Similar to the non-rotational von Karman dynamics, the Berger nonlinearity is not compact, and this requires some care in energy estimates.

Finally, what is perhaps the most challenging aspect of the analysis herein, is the difficulty involved in handling the trace terms associated to (HD) dynamics in the long-time behavior analysis. These terms include the standard linear terms - due to the principal biharmonic part-as well as "new" boundary terms arising due to the interaction of the boundary conditions, requisite multipliers, and the Berger nonlinearity. We must deal carefully with signed terms on the boundary (including imposing a geometric assumption on the domain), as well as utilize the sharp trace results on second order traces (as in [25]).

\subsubsection{Principal Contributions}

In what follows we address each of the points outlined above, as well as make additional contributions: 
- We show that a high (polynomial) degree of nonlinear damping can accommodate (at least in terms of well-posedness) the free-type boundary condition (FCD).

- We demonstrate asymptotic smoothness for the dynamical system associated with the nonrotational Berger dynamics under (HD) conditions. This utilizes a decomposition of Berger's nonlinearity, akin to the one utilized in the analogous result for non-rotational von Karman dynamics [14, pp. 496-497].

- We critically use what is in [19] in considering (HD) conditions, but we have a different treatment of trace terms (particularly in the use of the sharp estimates in [25]). This has the benefit of extending the approach in [19] to the non-rotational plates considered here.

- For the (HD) case, we are able to adapt the approach in [12] (corresponding to a semilinear wave equation with boundary damping) to construct the compact global attractor by directly obtaining estimates on the absorbing ball. This depends in a critical way on the special structure of the Berger nonlinearity (and does not seem possible for von Karman). We point out that, in some sense, we obtain a stronger result than in the analogous case for von Karman in [14.

Remark 1.9. The authors of [14] utilize additional assumptions on the damping to obtain a unique continuation result that provides a strict Lyapunov function for the dynamics. Then, they use an indirect result (for a gradient dynamical system, asymptotic smoothness implies the existence of a compact global attractor - see the Appendix, Theorem 6.1); this is to circumvent the need for direct estimation on the absorbing ball in the case of non-rotational von Karman dynamics.

- Most importantly, by addressing non-rotational Berger dynamics with boundary dissipation we provide results which seem to be conspicuously missing in the mathematical literature. As we have pointed out, the case of (HD) and (FCD) seem to be pathological, in that the analysis of the Berger dynamics is not subsumed by that of the von Karman.

\section{Abstract Plate Equations and Well-Posedness}

The issue of well-posedness of strong or finite energy solutions for nonlinear plates is recent. For a detailed and complete discussion of general, abstract plate equations the reader is referred to [14. For clarity, let us recall some definitions and results. The general model arising in nonlinear plate dynamics is:

$$
\left\{\begin{array}{l}
u_{t t}(t)+\mathcal{A} u(t)+\mathcal{A} G \bar{g}\left(G^{*} \mathcal{A} u_{t}(t)\right)=F\left(u(t), u_{t}(t)\right), \quad t>0 \\
u(0)=u_{0}, \quad u_{t}(0)=u_{1} .
\end{array}\right.
$$

To address the well-posedness for problem 2.1), we must give assumptions and properties of the operators.

Assumption 3. With reference to problem (2.1):

- Let $\mathcal{A}$ be a closed, linear, positive, self-adjoint operator acting on a Hilbert space $\mathcal{H}$, with $\mathscr{D}(\mathcal{A}) \subset$ $\mathcal{H}$. 
- Let $U$ be another Hilbert space, and $U_{0}$ be a reflexive Banach space, such that $U_{0} \subseteq U \subseteq U_{0}^{\prime}$. Additionally, assume that $\bar{g}: U_{0} \rightarrow U_{0}^{\prime}$ is a continuous mapping such that $\bar{g}(0)=0$ and

$$
\left\langle\bar{g}\left(v_{1}\right)-\bar{g}\left(v_{2}\right), v_{1}-v_{2}\right\rangle \geq 0, \quad \text { for all } v_{1}, v_{2} \in U_{0}
$$

where $\langle\cdot, \cdot\rangle$ denotes the scalar product on $U$, or the duality pairing between $U_{0}$ and $U_{0}^{\prime}$.

- The linear operator $G: U_{0}^{\prime} \rightarrow \mathcal{H}$ has that $\mathcal{A}^{1 / 2} G: U_{0}^{\prime} \rightarrow \mathcal{H}$ is bounded (or equivalently, $G^{*} \mathcal{A}: \mathscr{D}\left(\mathcal{A}^{1 / 2}\right) \rightarrow U_{0}$ is bounded, where the adjoint operator $G^{*}$ is defined by the relation $\left.\left\langle G^{*} u, v\right\rangle=\langle u, G v\rangle\right)$.

- Let $V$ be a Hilbert space satisfying $\mathscr{D}\left(\mathcal{A}^{1 / 2}\right) \subset V \subset \mathcal{H} \subset V^{\prime} \subset \mathscr{D}\left(\mathcal{A}^{1 / 2}\right)^{\prime}$ with all injections being continuous and dense. The nonlinear operator $F: \mathscr{D}\left(\mathcal{A}^{1 / 2}\right) \times V \rightarrow V^{\prime}$ is locally Lipschitz; that is:

$$
\left\|F\left(u_{1}, v_{1}\right)-F\left(u_{2}, v_{2}\right)\right\|_{V^{\prime}} \leq L(K)\left(\left\|\mathcal{A}^{1 / 2}\left(u_{1}-u_{2}\right)\right\|_{\mathcal{H}}+\left\|v_{1}-v_{2}\right\|_{V}\right)
$$

for all $\left(u_{i}, v_{i}\right) \in \mathscr{D}\left(\mathcal{A}^{1 / 2}\right) \times V$ such that $\left\|\mathcal{A}^{1 / 2} u_{i}\right\|_{\mathcal{H}},\left\|v_{i}\right\|_{V} \leq K$.

Remark 2.1. In concrete applications, the term $\mathcal{A} G \bar{g}\left(G^{*} \mathcal{A} u_{t}\right)$ models the boundary dissipation. Here, the operator $G$ typically represents a suitable Green's map, and the mapping $\bar{g}: U_{0} \rightarrow U_{0}^{\prime}$ is a Nemytskij-type operator (see Section 3 and 4).

If we rewrite problem 2.1] as a first order equation we have the equivalent problem

$$
\frac{d}{d t} \mathbf{u}(t)+\mathbb{A} \mathbf{u}(t)=\left[\begin{array}{c}
0 \\
F\left(u(t), u_{t}(t)\right)
\end{array}\right], \quad \mathbf{u}(0)=\mathbf{u}_{0} \equiv\left(u_{0}, u_{1}\right),
$$

where $\mathbf{u}(t)=\left(u(t), u_{t}(t)\right)$ and the operator $\mathbb{A}: \mathscr{D}\left(\mathcal{A}^{1 / 2}\right) \times V \rightarrow \mathscr{D}\left(\mathcal{A}^{1 / 2}\right) \times V$ is defined by

$$
\mathbb{A}=\left[\begin{array}{cc}
0 & -I \\
\mathcal{A} & \mathcal{A} G \bar{g} G^{*} \mathcal{A}
\end{array}\right]
$$

where $\mathscr{D}(\mathbb{A})$ consists of elements $(x, y) \in \mathscr{D}\left(\mathcal{A}^{1 / 2}\right) \times \mathscr{D}\left(\mathcal{A}^{1 / 2}\right)$ possessing the property

$$
\mathcal{A}\left(x+G \bar{g}\left(G^{*} \mathcal{A} y\right)\right) \in V^{\prime} .
$$

This structure of problem 2.1 leads the following definitions of strong and generalized solutions in this setting.

Definition 1. A function $u(t)$ is said to be a strong solution to 2.1] on a semi-interval $[0, T)$, iff

- $u(t) \in C\left([0, T) ; \mathscr{D}\left(\mathcal{A}^{1 / 2}\right)\right) \cap C^{1}([0, T) ; V)$

- $u \in W^{1,1}\left(a, b ; \mathscr{D}\left(\mathcal{A}^{1 / 2}\right)\right)$ and $u_{t} \in W^{1,1}(a, b ; V)$ for any $0<a<b<T$

- $\mathcal{A}\left[u(t)+G \bar{g}\left(G^{*} \mathcal{A} u_{t}(t)\right)\right] \in V^{\prime}$ for almost all $t \in[0, T]$

- Equation (2.1) is satisfied in $V^{\prime}$ for almost all $t \in[0, T]$

- The initial condition in (2.1) holds. 
The function $u(t)$ is a strong solution on $[0, T]$ if, in addition, we have that $\left(u(t), u_{t}(t)\right)$ is continuous at $t=T$. A function $u(t)$ is said to be a generalized solution to 2.1] on $[0, T]$, iff

- $u(t) \in C\left(0, T ; \mathscr{D}\left(\mathcal{A}^{1 / 2}\right)\right) \cap C^{1}(0, T ; V)$

- The initial condition in 2.1) holds.

- There exists sequences of strong solutions $\left\{u_{n}(t)\right\}$ to problem 2.1) defined on $[0, T]$ with initial data $\left(u_{0 n}, u_{1 n}\right)$ such that

$$
\lim _{n \rightarrow \infty} \max _{t \in[0, T]}\left\{\left\|u_{n t}(t)-u_{t}(t)\right\|_{V}+\left\|\mathcal{A}^{1 / 2}\left(u_{n}(t)-u(t)\right)\right\|_{\mathcal{H}}\right\}=0
$$

The function $u(t)$ is the generalized solution on a semi-interval $[0, T)$, if $u(t)$ is a generalized solution on each subinterval $\left[0, T^{\prime}\right] \subset[0, T)$.

The following theorem provides the existence and uniqueness of local and global solutions. This is the primary result of Section 2.4 in [14.

Theorem 2.1. Under Assumption [3, the following hold:

- Local strong solutions: For every $u_{0}, u_{1} \in \mathscr{D}\left(\mathcal{A}^{1 / 2}\right)$ such that $\mathcal{A}\left[u_{0}+G \bar{g}\left(G^{*} \mathcal{A} u_{1}\right)\right] \in V^{\prime}$ there exists $t_{\max }>0$ and unique strong solution such that $\left(u, u_{t}\right) \in C\left(\left[0, t_{\max }\right) ; \mathscr{D}\left(\mathcal{A}^{1 / 2}\right) \times V\right)$.

- Local generalized solutions: If $\left(u_{0}, u_{1}\right) \in \overline{\mathscr{D}(\mathbb{A})} \subseteq \mathscr{D}\left(\mathcal{A}^{1 / 2}\right) \times V$, where $\overline{\mathscr{D}(\mathbb{A})}$ is the closure of $\mathscr{D}(\mathbb{A})$ in $\mathscr{D}\left(\mathcal{A}^{1 / 2}\right) \times V$. Then there exists $t_{\max }>0$ and unique generalized solution such that $\left(u, u_{t}\right) \in C\left(\left[0, t_{\max }\right) ; \mathscr{D}\left(\mathcal{A}^{1 / 2}\right) \times V\right)$.

- Global solutions: If, in addition, strong (or generalized) solutions satisfy

$$
\sup _{t \in\left[0, t_{*}\right]}\left\{\left\|\mathcal{A}^{1 / 2} u(t)\right\|_{\mathcal{H}}+\left\|u_{t}(t)\right\|_{V}\right\} \leq M\left(t_{*}, u_{0}, u_{1}\right)
$$

for every existence semi-interval $\left[0, t_{*}\right]$, then the local solutions referred to above are global, which is to say $t_{\max }=\infty$.

\section{Berger Model with (FCD) Conditions}

\subsection{Well-posedness - Proof of Theorem 1.1}

We consider problem (1.1) with (FCD) boundary conditions. The proof of Theorem 1.1 is based on the application of the abstract setup above and Theorem 2.1

\subsubsection{Application of Abstract Setup and Local Well-posedness}

Firstly, we need to modify the abstract model given above to fit our case. For the functional setup, we introduce the following spaces and operators:

- $\mathcal{H}=V \equiv L^{2}(\Omega), \quad U \equiv L^{2}\left(\Gamma_{1}\right) \times L^{2}\left(\Gamma_{1}\right)$, 
- $U_{0} \equiv H^{1 / 2}\left(\Gamma_{1}\right) \times H^{3 / 2}\left(\Gamma_{1}\right), \quad U_{0}^{\prime}=H^{-1 / 2}\left(\Gamma_{1}\right) \times H^{-3 / 2}\left(\Gamma_{1}\right)$,

- $\mathcal{A} u \equiv \Delta^{2} u, u \in \mathscr{D}(\mathcal{A})$, where

$$
\mathscr{D}(\mathcal{A}) \equiv\left\{\begin{array}{c}
u=\nabla u=0 \text { on } \Gamma_{0} \\
u \in H^{4}(\Omega): \partial_{\nu} \Delta u+(1-\mu) B_{2} u-\mu_{1} u=0 \text { on } \Gamma_{1} \\
\Delta u+(1-\mu) B_{1} u=0 \text { on } \Gamma_{1}
\end{array}\right\}
$$

- $V^{\prime}=L^{2}(\Omega)$ and $\mathscr{D}\left(\mathcal{A}^{1 / 2}\right)=H_{\Gamma_{0}}^{2}(\Omega)$ which is given in Section 1.3.

- $F(u, v)=-f_{B}(u)+p$, with $f_{B}(u)=\left(\gamma-\|\nabla u\|^{2}\right) \Delta u$,

- $G: U_{0}^{\prime} \rightarrow \mathcal{H}, G\left(g_{1}, g_{2}\right) \equiv G_{1}\left(g_{1}\right)-G_{2}\left(g_{2}\right)$ where $G_{i}, i=1,2$, are defined by

$$
G_{1}(v) \equiv u \text { iff }\left\{\begin{array}{c}
\Delta^{2} u=0 \text { in } \Omega, u=\nabla u=0 \text { on } \Gamma_{0} \\
\Delta u+(1-\mu) B_{1} u=v \text { on } \Gamma_{1} \\
\partial_{\nu} \Delta u+(1-\mu) B_{2} u=\mu_{1} u \text { on } \Gamma_{1},
\end{array}\right.
$$

and

$$
G_{2}(v) \equiv u \text { iff }\left\{\begin{array}{c}
\Delta^{2} u=0 \text { in } \Omega, u=\nabla u=0 \text { on } \Gamma_{0} \\
\Delta u+(1-\mu) B_{1} u=0 \text { on } \Gamma_{1} \\
\partial_{\nu} \Delta u+(1-\mu) B_{2} u=\mu_{1} u+v \text { on } \Gamma_{1} .
\end{array}\right.
$$

- The mapping $\bar{g}: U_{0} \rightarrow U_{0}^{\prime}$ has the form

$$
\bar{g}:\left(v_{1}, v_{2}\right) \rightarrow\left(g_{1}\left(v_{1}\right), g_{2}\left(v_{2}\right)\right), \quad\left(v_{1}, v_{2}\right) \in U_{0}
$$

With the above notation, taking into account that in our case: $g_{1}=0$ and $g_{2}(v)=|v|{ }^{6} v, G\left(g_{1}, g_{2}\right) \equiv$ $-G_{2}\left(g_{2}\right)$. We reduce the equation in (1.1) to the following form

$$
u_{t t}+\mathcal{A}\left[u-G_{2}\left(\left.\left[\left|u_{t}\right|{ }^{6} u_{t}\right]\right|_{\Gamma_{1}}\right)\right]=F\left(u(t), u_{t}(t)\right), \quad t>0,
$$

or equivalently,

$$
u_{t t}+\mathcal{A}\left[u+G \bar{g}\left(G^{*} \mathcal{A} u_{t}\right)\right]=F\left(u(t), u_{t}(t)\right), \quad t>0 .
$$

Now, before giving the well-posedness result for (3.1), we verify that the conditions in Assumption 3 are satisfied.

Lemma 3.1. With reference to 3.1, operators $\mathcal{A}, G$ and $F$ introduced above comply with the requirements of Assumption 3 .

Proof. By definition of $\mathcal{A}$ as above, $\mathcal{A}$ is closed, linear, positive, self-adjoint, densely defined on $\mathcal{H}$ with $\mathscr{D}\left(\mathcal{A}^{1 / 2}\right)=H_{\Gamma_{0}}^{2}(\Omega)$, and we have dense, continuous injections:

$$
\mathscr{D}\left(\mathcal{A}^{1 / 2}\right) \subset V=\mathcal{H}=V^{\prime} \subset \mathscr{D}\left(\mathcal{A}^{1 / 2}\right)^{\prime}=\left[H_{\Gamma_{0}}^{2}(\Omega)\right]^{\prime} .
$$

The Nemytskij operator

$$
\left(g_{1}, g_{2}\right)=\left(0, g_{2}\right): U_{0} \rightarrow U_{0}^{\prime}
$$


is monotone [14, 31] and continuous, since $H^{3 / 2}\left(\Gamma_{1}\right) \subset C\left(\Gamma_{1}\right)$ by the Sobolev embeddings.

The requirement on $G$ in Assumption 3 reduces the fact that

$$
G^{*} \mathcal{A}^{1 / 2}: \mathcal{H} \rightarrow U_{0} \equiv H^{1 / 2}\left(\Gamma_{1}\right) \times H^{3 / 2}\left(\Gamma_{1}\right)
$$

is bounded. An application of Green's theorem with the calculations given in [14, Section 3.2.2] yield that the mappings

$$
\begin{aligned}
G_{1}: L^{2}\left(\Gamma_{1}\right) & \rightarrow \mathscr{D}\left(\mathcal{A}^{5 / 8-\epsilon}\right), \quad G_{2}: L^{2}\left(\Gamma_{1}\right) \rightarrow \mathscr{D}\left(\mathcal{A}^{7 / 8-\epsilon}\right) \\
G_{1}^{*} \mathcal{A}: \mathscr{D}\left(\mathcal{A}^{1 / 2}\right) & \rightarrow H^{1 / 2}\left(\Gamma_{1}\right), \quad G_{2}^{*} \mathcal{A}: \mathscr{D}\left(\mathcal{A}^{1 / 2}\right) \rightarrow H^{3 / 2}\left(\Gamma_{1}\right)
\end{aligned}
$$

are bounded and for every $u \in \mathscr{D}\left(\mathcal{A}^{3 / 8+\epsilon}\right) \subset H^{3 / 2+4 \epsilon}(\Omega)$. We then have that

$$
G^{*} \mathcal{A} u=\left(G_{1}^{*} \mathcal{A} u,-G_{2}^{*} \mathcal{A} u\right)=\left(\left.\partial_{\nu} u\right|_{\Gamma_{1}},\left.u\right|_{\Gamma_{1}}\right) .
$$

By the last equality, 3.2 translates into the fact that

$$
u \rightarrow G^{*} \mathcal{A}^{1 / 2} u=\left(\partial_{\nu} \mathcal{A}^{-1 / 2} u, \mathcal{A}^{-1 / 2} u\right)
$$

is bounded from $L^{2}(\Omega)$ into $H^{1 / 2}\left(\Gamma_{1}\right) \times H^{3 / 2}\left(\Gamma_{1}\right)$. This follows from the identification $\mathscr{D}\left(\mathcal{A}^{1 / 2}\right)=$ $H_{\Gamma_{0}}^{2}(\Omega)$, and the trace theorem, which imply that the maps

$$
\partial_{\nu}(\cdot): H^{2}(\Omega) \rightarrow H^{1 / 2}(\Gamma),\left.\quad(\cdot)\right|_{\Gamma}: H^{2}(\Omega) \rightarrow H^{3 / 2}(\Gamma)
$$

are bounded. The validity of requirement on $G$ is thus observed.

Now, we need to verify that the operator $F: \mathscr{D}\left(\mathcal{A}^{1 / 2}\right) \times V \rightarrow V^{\prime}(=V)$, defined by

$$
F(u, v)=-f_{B}(u)+p,
$$

is locally Lipschitz. Let $u, w \in \mathscr{D}\left(\mathcal{A}^{1 / 2}\right)$ with $\left\|A^{1 / 2} u\right\|_{\mathcal{H}},\left\|A^{1 / 2} w\right\|_{\mathcal{H}} \leq R$, where the bilinear form $a(\cdot, \cdot)$ plays a special role for the norm in $\mathscr{H}$ under $(\mathrm{FCD})$ and is equivalent to $\|\Delta \cdot\|_{0}$ for (HD)

$$
\left\|f_{B}(u)-f_{B}(w)\right\|_{V} \leq C\|u-w\|_{\mathscr{D}\left(\mathcal{A}^{1 / 2}\right)}+\|\| \nabla u\left\|^{2} \Delta u-\right\| \nabla w\left\|^{2} \Delta w\right\| .
$$

Now,

$$
\begin{aligned}
\|\| \nabla u\left\|^{2} \Delta u-\right\| \nabla w\left\|^{2} \Delta w\right\| & \leq\|\| \nabla u\left\|^{2} \Delta u-\right\| \nabla u\left\|^{2} \Delta w+\right\| \nabla u\left\|^{2} \Delta w-\right\| \nabla w\left\|^{2} \Delta w\right\| \\
& \leq\|\| \nabla u\left\|^{2} \Delta(u-w)+\Delta w\left(\|\nabla u\|^{2}-\|\nabla w\|^{2}\right)\right\| \\
& \leq C\|u\|_{1, \Omega}^{2}\|u-w\|_{2, \Omega}+C\|w\|_{2, \Omega}(\|\nabla u\|+\|\nabla w\|)(\|\nabla u\|-\|\nabla w\|) \\
& \leq C\|u\|_{1, \Omega}^{2}\|u-w\|_{2, \Omega}+C\|w\|_{2, \Omega}(\|\nabla u\|+\|\nabla w\|)\left(\|u-w\|_{1, \Omega}\right) \\
& \leq C(R)\|u-w\|_{\mathscr{D}\left(A^{1 / 2}\right)},
\end{aligned}
$$

where we have freely used the Poincare's inequality, the equivalence $H^{2}(\Omega) \equiv \mathscr{D}\left(A^{1 / 2}\right)$, and Sobolev

\footnotetext{
${ }^{5}$ For (HD), this follows from the well-posedness of the biharmonic problem associated to the these boundary conditions.
} 
embeddings. The locally Lipschitz property for $F$ is thus observed.

Moreover, for initial data $\left(u_{0}, u_{1}\right) \in \mathscr{H}$, since the set

$$
\mathcal{L}=\left(H_{\Gamma_{0}}^{2} \cap H^{4}\right)(\Omega) \times C_{0}^{\infty}(\Omega)
$$

is dense in $\mathscr{H}$ and belongs to the domain of corresponding operator $\mathbb{A}$ given in 2.2 , the application of Theorem 2.1 gives the local (in time) existence and uniqueness of generalized solutions.

\section{A Priori Bound and Global Well-posedness-Completion of proof of Theorem 1.1}

Having obtained local generalized solutions, our aim is to obtain global a priori estimates for these solutions. We note that our computations are first performed on strong solutions, and then extended via density to $\mathscr{H}$ in the limit. Our starting point is the energy relation 1.9 , which follows via integration by parts for strong solutions and remains valid for generalized solutions. It can easily be shown [10] that there exists positive constants $c_{0}, c_{1}$ and $C$ such that

$$
c_{0} \mathscr{E}\left(u, u_{t}\right)-C \leq E\left(u, u_{t}\right) \leq c_{1} \mathscr{E}\left(u, u_{t}\right)+C
$$

for all generalized solutions $\left(u, u_{t}\right) \in \mathscr{H}\left[{ }^{6}\right.$ Now, using $(3.3)$ in the energy identity $(1.9)$, we have

$$
E(t)+\int_{0}^{t} \int_{\Gamma_{1}}\left|u_{t}\right|^{8}+\int_{0}^{t} \int_{\Gamma_{1}}\left(\gamma-\|\nabla u\|^{2}\right)\left(\partial_{\nu} u\right) u_{t} \leq E(0)+C
$$

Dealing with the non-dissipative term above requires some calculation. By the Hölder inequality we have

$$
\left|\int_{0}^{t} \int_{\Gamma_{1}}\left(\gamma-\|\nabla u\|^{2}\right)\left(\partial_{\nu} u\right)\left(u_{t}\right)\right| \leq C\left\{\int_{0}^{t} \gamma\left\|\partial_{\nu} u\right\|_{L^{2}\left(\Gamma_{1}\right)}\left\|u_{t}\right\|_{L^{2}\left(\Gamma_{1}\right)}+\int_{0}^{t}\|\nabla u\|^{2}\left\|\partial_{\nu} u\right\|_{L^{2}\left(\Gamma_{1}\right)}\left\|u_{t}\right\|_{L^{2}\left(\Gamma_{1}\right)}\right\} .
$$

Now, applying the trace moment inequality for $\partial_{\nu} u,[9$, Theorem 1.6.6] we have

$$
\left|\int_{0}^{t} \int_{\Gamma_{1}}\left(\gamma-\|\nabla u\|^{2}\right)\left(\partial_{\nu} u\right) u_{t}\right| \leq C\left\{\int_{0}^{t} \gamma\|u\|_{2}^{1 / 2}\|\nabla u\|^{1 / 2}\left\|u_{t}\right\|_{L^{2}\left(\Gamma_{1}\right)}+\int_{0}^{t}\|u\|_{2}^{1 / 2}\|\nabla u\|^{5 / 2}\left\|u_{t}\right\|_{L^{2}\left(\Gamma_{1}\right)}\right\} .
$$

We note the equivalence of $a(\cdot, \cdot)$ and $\|\cdot\|_{2}$. We then use Young's inequality for the first integral on the RHS, with powers $2,4,8$, and 8 , so $(1 / 2)+(1 / 4)+(1 / 8)+(1 / 8)=1$. For the second integral of RHS, with powers $4,8 / 5,8$, where $(1 / 4)+(5 / 8)+(1 / 8)=1$, we obtain

$$
\left|\int_{0}^{t} \int_{\Gamma_{1}}\left(\gamma-\|\nabla u\|^{2}\right)\left(\partial_{\nu} u\right) u_{t}\right| \leq C_{\epsilon} \gamma^{2} t+C_{\epsilon} \int_{0}^{t} E(s) d s+\epsilon \int_{0}^{t}\left\|u_{t}\right\|_{L^{2}\left(\Gamma_{1}\right)}^{8} .
$$

This yields

\footnotetext{
${ }^{6}$ For more details, see the discussion preceding 4.3 .
} 


$$
E(t) \leq C(\gamma, t, E(0))+C \int_{0}^{t} E(s) d s .
$$

An application of Gronwall's inequality gives:

$$
E(t) \leq C(T), \quad \text { for all } t \in[0, T]
$$

This implies the global existence of strong (and thus generalized) solutions to equation (3.1). Since these solutions also solve the original problem - 1.1 with (FCD) boundary conditions - the proof of Theorem 1.1 is now completed.

Remark 3.1. At the present time, we do not see how the global well-posedness of solutions can be established in the absence of such highly nonlinear damping. As discussed above, this is an indication that the dynamical systems properties of solutions does not follow readily from the equations via standard techniques used at the finite-energy level. Without highly nonlinear damping active on the boundary, we may not have control of the non-dissipative term

$$
\int_{0}^{t}\left(\gamma-\|\nabla u\|^{2}\right) \int_{\Gamma_{1}}\left(\partial_{\nu} u\right) u_{t} d \Gamma d t
$$

Additionally, since we have no control over $E(t)$ as $T \rightarrow+\infty$, long-time behavior analysis is not viable.

\section{Berger Model with (HD) Conditions}

In this section we consider problem (1.1) with (HD) boundary conditions.

\subsection{Well-posedness-Proof of Theorem 1.2}

Similar to Section 3, the proof of Theorem 1.2 is based on the abstract setup given in Section 2 and the application of Theorem 2.1. We adapt the abstract model 2.1 to this case.

\subsubsection{Application of Abstract Setup and Verification of Hypotheses}

We introduce the following spaces and operators:

- $\mathcal{H}=L^{2}(\Omega), \quad U \equiv L^{2}(\Gamma)$

- $U_{0} \equiv H^{1 / 2}(\Gamma) \quad U_{0}^{\prime}=H^{-1 / 2}(\Gamma)$

- $\mathcal{A} u \equiv \Delta^{2} u, u \in \mathscr{D}(\mathcal{A})$, where

$$
\mathscr{D}(\mathcal{A}) \equiv\left\{u \text { in }\left(H^{4} \cap H_{0}^{1}\right)(\Omega): \Delta u=0 \text { on } \Gamma\right\}
$$

- $V^{\prime}=V=L^{2}(\Omega)$ and $\mathscr{D}\left(\mathcal{A}^{1 / 2}\right)=\left(H_{0}^{1} \cap H^{2}\right)(\Omega)$. 
- $F(u, v)=-f_{B}(u)+p$ where $f_{B}(u)=\left(\gamma-\|\nabla u\|^{2}\right) \Delta u$.

- $G: L^{2}(\Gamma) \rightarrow L^{2}(\Omega)$ denotes a biharmonic extension of the boundary values defined on $\Gamma$. That is:

$$
G(v) \equiv u \operatorname{iff}\left\{\begin{array}{c}
\Delta^{2} u=0 \text { in } \Omega \\
u=0 \text { on } \Gamma \\
\Delta u=v \text { on } \Gamma
\end{array}\right.
$$

- The mapping $\bar{g}: U_{0} \rightarrow U_{0}^{\prime}$ is determined by the function $D(\cdot)$ according to the formula

$$
(\bar{g}(v), w)_{U_{0}, U_{0}^{\prime}}=\int_{\Gamma} D(v) w d \Gamma \quad v, w \in U_{0} .
$$

We note that the application of Green's formula gives that $G^{*} \mathcal{A}=\left.\frac{\partial}{\partial \nu}\right|_{\Gamma}$, and hence $\operatorname{ker}\left[G^{*} \mathcal{A}\right] \cap$ $\mathscr{D}\left(\mathcal{A}^{1 / 2}\right)$ contains $H_{0}^{2}(\Omega)$ and is dense in $L^{2}(\Omega)$. This implies that for all initial data $\left(u_{0}, u_{1}\right) \in \mathscr{H}$ the set $\mathscr{D}(\mathcal{A}) \times\left(\operatorname{ker}\left[G^{*} \mathcal{A}\right] \cap \mathscr{D}\left(\mathcal{A}^{1 / 2}\right)\right)$ is dense in $\mathscr{H}$ and belongs to the domain of the corresponding operator $\mathbb{A}$.

In line with the above setup, the verification of the requirements of Assumption 3 follows as the case of (FCD) damping. For more details the reader is referred to [14, Section 4.2]. The proof of Theorem 1.2 is obtained by the application of Theorem 2.1 via the density argument above.

\subsection{Long Time Behavior Under (HD) — Proof of Theorem 1.3}

This section is devoted to study the long time behavior of dynamical system generated by the solution of (1.1) taken with (HD) boundary conditions. Our principal goal is to prove Theorem 1.3, the existence of a compact global attractor for the dynamics.

We utilize the notations consistent with the case of (FCD), but now specified to the case of (HD): $E(t)=\frac{1}{2}\left\{\left\|u_{t}\right\|^{2}+\|\Delta u\|^{2}\right\}$, as well as the identical definitions for $\widehat{E}$ and $\mathscr{E}$ from before.

As in the proof of [14, Lemma 1.5.4], for all $\left(u, u_{t}\right) \in \mathscr{H}=\left(H^{2} \cap H_{0}^{1}\right)(\Omega) \times L^{2}(\Omega)$, and any $\epsilon>0$, it is straightforward to show the potential energy bound (as we will refer to it below):

$$
\left|\frac{\gamma}{2}\|\nabla u(t)\|^{2}+(p, u)\right| \leq \epsilon\left[\|\Delta u(t)\|^{2}+\frac{1}{2}\|\nabla u(t)\|^{4}\right]+M(\epsilon, \gamma, p) .
$$

Remark 4.1. In fact, as in [14, the key bound which implies 4.1] is

$$
\|u\|_{2-\eta}^{2} \leq \epsilon\left[\|\Delta u(t)\|^{2}+\frac{1}{2}\|\nabla u(t)\|^{4}\right]+M(\epsilon), \quad \eta, \epsilon>0
$$

This also yields the energetic bounds

$$
c_{0} \widehat{E}\left(u, u_{t}\right)-C \leq \mathscr{E}\left(u, u_{t}\right) \leq c_{1} \widehat{E}\left(u, u_{t}\right)+C,
$$

for some $c_{0}, c_{1}, C>0$ depending on $p$ and $\gamma$. Accordingly, we introduce more notation for the study of 
long-time behavior (following [12]):

$$
0 \leq \mathscr{E}_{M}(t) \equiv \mathscr{E}(t)+M
$$

where $M=M(\epsilon, \gamma, p)$ is the constant given in (4.1).

\subsubsection{Overview of the Proof}

Proof of Theorem 1.3 (which gives the existence of a compact global attractor for $\left(S_{H}, \mathscr{H}\right)$ ) follows from the application of the abstract Theorem 6.1 (in the Appendix). Utilizing Theorem 6.1 requires showing that the dynamical system generated by solutions to (1.1) taken with (HD) conditions is dissipative (i.e., possess a bounded absorbing set), and has the asymptotic smoothness property. Thus, to prove Theorem 1.3 , we will first present the dissipativity analysis of 1.1 in Section 4.2.2 We will utilize energy methods to show the existence of the absorbing ball that attracts every trajectory after a certain time. This will be given as Theorem 4.1. Then, in Theorem 4.4 the asymptotic smoothness property of the dynamical system is given. Its proof follows through several steps in Section 4.2.3. With these two results, the existence of a compact global attractor for $\left(S_{H}, \mathscr{H}\right)$ will be demonstrated.

Remark 4.2. We will show an explicit bound on the size of the absorbing ball. We note that this is in contrast to the approach taken in [14] for the non-rotational von Karman plate with hinged-type boundary dissipation; there, the dynamics (under additional assumptions, including requisite frictional damping in the interior) are shown to be gradient, and thus the existence of a compact global attractor (roughly) follows from the asymptotic smoothness property (see Theorem 6.2 in the Appendix). Also see the previous discussion following Theorem 1.3 .

\subsubsection{Explicit Estimates on Absorbing Ball}

In this section, as the first step of the proof of Theorem 1.3 , we show the dissipativity of the dynamical system corresponding to generalized solutions of (1.1) with (HD) boundary conditions. To this end, we give an explicit estimate on the absorbing ball for which we utilize multiplier techniques and sharp trace results for the linear plate equation with a given RHS [25]. We will show the following theorem:

Theorem 4.1. Let Assumption 1 and 2 hold. Then there exists an absorbing set $\mathscr{B} \subset \mathscr{H}$ for generalized solutions to (1.1) taken with (HD) boundary conditions. This is to say: for all $R_{0}>0$ and initial data $\left(u_{0}, u_{1}\right) \in \mathscr{H}$ with $\left\|\left(u_{0}, u_{1}\right)\right\|_{\mathscr{H}} \leq R_{0}$, there exists a $t_{0}=t\left(R_{0}\right)$ such that $\left(u(t), u_{t}(t)\right) \in \mathscr{B}$ for $t \geq t_{0}$.

In order to prove this central theorem, the main technical ingredient will be the following "observability" estimate, stated below.

Lemma 4.2. Let Assumption 2 is in force. Let $T>0$ and $0<\alpha<T / 2$. Then any solution to (1.1) taken with (HD) boundary conditions satisfies the following estimate:

$$
\begin{gathered}
(T-2 \alpha) \widehat{E}(T) \leq C \widehat{E}(0)+C(T)\left\|D\left(\partial_{\nu} u_{t}\right)\right\|_{L^{2}\left(0, T ; L^{2}(\Gamma)\right)}^{2}\left[\int_{0}^{T}(\widehat{E}(\tau))^{2} d \tau\right] \\
+C(T)\left[\left\|D\left(\partial_{\nu} u_{t}\right)\right\|_{L^{2}\left(0, T ; L^{2}(\Gamma)\right)}^{2}+\left\|\partial_{\nu} u_{t}\right\|_{L^{2}\left(0, T ; L^{2}(\Gamma)\right)}^{2}\right]+C(T) .
\end{gathered}
$$


Proof of Lemma 4.2. Now, let $u$ be a generalized solution to (1.1) taken with (HD) boundary conditions. We begin with the equipartition multiplier $u$ and the flux multiplier $h \cdot \nabla u$, where $h(\mathbf{x})=\mathbf{x}-\mathbf{x}_{0}$ for some appropriately chosen $\mathbf{x}_{0} \in \mathbb{R}^{2}$ (satisfying Assumption 2). Multiplying (1.1) by $u$ and $h \cdot \nabla u$, respectively, integrating over the space-time cylinder, and using Green's theorem we have

$$
\int_{0}^{T}\left\{\|\Delta u\|^{2}+\|\nabla u\|^{4}-\left\|u_{t}\right\|^{2}\right\}+\int_{0}^{T} \int_{\Gamma} D\left(\partial_{\nu} u_{t}\right)\left(\partial_{\nu} u\right)=-\left.\left(u_{t}, u\right)\right|_{0} ^{T}+\int_{0}^{T}\left\{(p, u)+\gamma\|\nabla u\|^{2}\right\},
$$

and

$$
\begin{aligned}
\int_{0}^{T} & \left\{\|\Delta u\|^{2}+\left\|u_{t}\right\|^{2}\right\}+\int_{0}^{T} \int_{\Gamma}\left\{\frac{1}{2}|\Delta u|^{2}(h \cdot \nu)+\partial_{\nu}(\Delta u)(h \cdot \nabla u)-(\Delta u) \partial_{\nu}(h \cdot \nabla u)\right\} \\
& +\frac{1}{2} \int_{0}^{T}\left(\gamma-\|\nabla u\|^{2}\right) \int_{\Gamma}(h \cdot \nu)\left|\partial_{\nu} u\right|^{2}=-\left.\left(u_{t}, h \cdot \nabla u\right)\right|_{0} ^{T}+\int_{0}^{T}(p, h \cdot \nabla u) .
\end{aligned}
$$

We have used the facts:

$$
\begin{aligned}
\left(\left(\gamma-\|\nabla u\|^{2}\right) \Delta u, h \cdot \nabla u\right) & =\left(\|\nabla u\|^{2}-\gamma\right)(\nabla u, \nabla(h \cdot \nabla u))+\int_{0}^{T}\left[\gamma-\|\nabla u\|^{2}\right] \int_{\Gamma}\left(\partial_{\nu} u\right)(h \cdot \nabla u) \\
(\nabla u, \nabla(h \cdot \nabla u)) & =\frac{1}{2} \operatorname{div}\left(h|\nabla u|^{2}\right) \quad \text { and } \quad h \cdot \nabla u=(h \cdot \nu) \partial_{\nu} u \text { on } \Gamma,
\end{aligned}
$$

owing to the divergence theorem, and the zero Dirichlet condition on the whole of $\Gamma$ (for (HD)). Now, taking a suitable combination of (4.6) and (4.7) we obtain:

$$
\begin{aligned}
\int_{0}^{T}\left\{3\|\Delta u\|^{2}\right. & \left.+\|\nabla u\|^{4}+\left\|u_{t}\right\|^{2}-\gamma\|\nabla u\|^{2}\right\}+\int_{0}^{T}\left(\gamma-\|\nabla u\|^{2}\right) \int_{\Gamma}(h \cdot \nu)\left|\partial_{\nu} u\right|^{2} \\
= & -\left\{\left.\left(u_{t}, u\right)\right|_{0} ^{T}+\left.2\left(u_{t}, h \cdot \nabla u\right)\right|_{0} ^{T}\right\}+\int_{0}^{T}(p, u)+2 \int_{0}^{T}(p, h \cdot \nabla u)-\int_{0}^{T} \int_{\Gamma} D\left(\partial_{\nu} u_{t}\right)\left(\partial_{\nu} u\right) \\
& +\int_{0}^{T} \int_{\Gamma}\left\{2(\Delta u) \partial_{\nu}(h \cdot \nabla u)-(h \cdot \nu)|\Delta u|^{2}-2 \partial_{\nu}(\Delta u)(h \cdot \nabla u)\right\} .
\end{aligned}
$$

Using the bound on the nonlinear potential energy (4.1) and reorganizing the terms above, we have a preliminary estimate:

$$
\begin{aligned}
c \int_{0}^{T} \widehat{E}(\tau) \leq & \\
(\mathbf{T} 1) & -\left\{\left.\left(u_{t}, u\right)\right|_{0} ^{T}+\left.2\left(u_{t}, h \cdot \nabla u\right)\right|_{0} ^{T}\right\}+\int_{0}^{T}(p, u)+2 \int_{0}^{T}(p, h \cdot \nabla u)+C(\gamma) \cdot T \\
(\mathbf{T 2}) & -2 \int_{0}^{T} \int_{\Gamma} \partial_{\nu}(\Delta u)(h \cdot \nabla u)-\int_{0}^{T}\left(\gamma-|| \nabla u \|^{2}\right) \int_{\Gamma}(h \cdot \nu)\left|\partial_{\nu} u\right|^{2} \\
(\mathbf{T} 3) & -\int_{0}^{T} \int_{\Gamma} D\left(\partial_{\nu} u_{t}\right)\left(\partial_{\nu} u\right)+\int_{0}^{T} \int_{\Gamma} 2(\Delta u) \partial_{\nu}(h \cdot \nabla u)-\int_{0}^{T} \int_{\Gamma}(h \cdot \nu)|\Delta u|^{2}
\end{aligned}
$$


Firstly, by 4.1 and Young's inequality, we observe

$$
|(\mathbf{T} 1)| \leq C \widehat{E}(0)+\epsilon\left[\widehat{E}(T)+\int_{0}^{T} \widehat{E}(\tau) d \tau\right]+C(p, \epsilon, \gamma) \cdot T .
$$

Now, employing the geometric Assumption 2 on (T2)-(T3), dropping the negatively signed terms on the RHS, combining our computations thus far and reorganizing, we obtain the following intermediate relation:

$$
\begin{aligned}
c \int_{0}^{T} \widehat{E}(\tau) \leq & C \widehat{E}(0)+\epsilon\left[\widehat{E}(T)+\int_{0}^{T} \widehat{E}(\tau)\right]+C(p, \epsilon, \gamma) \cdot T \\
& -2 \int_{0}^{T} \int_{\Gamma} \partial_{\nu}(\Delta u)(h \cdot \nabla u)-\int_{0}^{T}\left(\gamma-\|\nabla u\|^{2}\right) \int_{\Gamma}(h \cdot \nu)\left|\partial_{\nu} u\right|^{2} \\
& -\int_{0}^{T} \int_{\Gamma} D\left(\partial_{\nu} u_{t}\right)\left(\partial_{\nu} u\right)+\int_{0}^{T} \int_{\Gamma} 2(\Delta u) \partial_{\nu}(h \cdot \nabla u) .
\end{aligned}
$$

In order to estimate the RHS of the above inequality - which involves higher order trace terms - we follow the analysis of [19] (which itself critically relies on Theorem 4.3 from [25] given below). However, we approach the higher order trace term involving $\partial_{\nu}(\Delta u)$ in a more straightforward way which, ultimately, benefits our analysis and fundamentally exploits the structure of the Berger nonlinearity to obtain cancellations.

Now, let the operator $A=\Delta$, acting on $L^{2}(\Omega)$ with domain $H^{2}(\Omega) \cap H_{0}^{1}(\Omega)$, and let $D_{L}$ be the associated Dirichlet "lift" map defined by

$$
D_{L} g=f \in L^{2}(\Omega) \Longleftrightarrow \Delta f=0 \text { in } \Omega \text { and } f=g \text { on } \Gamma \text {. }
$$

Let $F \equiv\left\{-f_{B}(u)+p\right\}$. Accounting for boundary conditions, the operator representation of 1.1 is then

$$
u_{t t}+A\left[A u-D_{L}\left(\left.\Delta u\right|_{\Gamma}\right)\right]=F .
$$

Applying $A^{-1}$ to 4.12 (justified on strong solutions, and a posteriori on generalized solutions via the corresponding estimate) we obtain

$$
\Delta u=A u=-A^{-1} u_{t t}+D_{L}\left(\left.\Delta u\right|_{\Gamma}\right)+A^{-1} F \text { in } \mathscr{D}^{\prime}(\Omega) .
$$

Now, taking the normal derivative of both sides of above equality, multiplying by $h \cdot \nabla u$, integrating over $[0, T] \times \Gamma$, and reading off from the equation we have the relation

$$
\begin{aligned}
\int_{0}^{T} \int_{\Gamma} \partial_{\nu}(\Delta u)(h \cdot \nabla u)= & -\int_{0}^{T} \int_{\Gamma} \partial_{\nu}\left(A^{-1} u_{t t}\right)(h \cdot \nabla u)+\int_{0}^{T} \int_{\Gamma} \partial_{\nu} D_{L}\left(\left.\Delta u\right|_{\Gamma}\right)(h \cdot \nabla u) \\
& +\int_{0}^{T} \int_{\Gamma}\left(\partial_{\nu} A^{-1} F\right)(h \cdot \nabla u) .
\end{aligned}
$$

In order to estimate each term of the RHS of 4.13 we use the approach in [19]. The following is the critical step which allows our approach to the higher order trace terms to obtain. This relies critically 
on the structure of the Berger nonlinearity. If we note that

$$
\begin{aligned}
A^{-1} F & =\left(\Delta_{D_{L}}\right)^{-1}\left[\|\nabla u\|^{2}-\gamma\right] \Delta u+A^{-1} p \\
& =\left[\|\nabla u\|^{2}-\gamma\right] u+A^{-1} p,
\end{aligned}
$$

and again $h \cdot \nabla u=(h \cdot \nu) \partial_{\nu} u$, since $u=0$ on $\Gamma$; we have:

$$
\begin{aligned}
-2 \int_{0}^{T} & \int_{\Gamma} \partial_{\nu}(\Delta u)(h \cdot \nabla u)-\int_{0}^{T}\left(\gamma-\|\nabla u\|^{2}\right) \int_{\Gamma}(h \cdot \nu)\left|\partial_{\nu} u\right|^{2} \\
= & 2 \int_{0}^{T} \int_{\Gamma} \partial_{\nu}\left(A^{-1} u_{t t}\right)(h \cdot \nabla u)-2 \int_{0}^{T} \int_{\Gamma} \partial_{\nu} D_{L}\left(\left.\Delta u\right|_{\Gamma}\right)(h \cdot \nabla u) \\
& +2 \int_{0}^{T}\left(\gamma-\|\nabla u\|^{2}\right) \int_{\Gamma}(h \cdot \nu)\left(\partial_{\nu} u\right)^{2}-2 \int_{0}^{T} \int_{\Gamma} \partial_{\nu}\left(A^{-1} p(\mathbf{x})\right)(h \cdot \nabla u) \\
& -\int_{0}^{T}\left(\gamma-\|\nabla u\|^{2}\right) \int_{\Gamma}(h \cdot \nu)\left|\partial_{\nu} u\right|^{2}
\end{aligned}
$$

Remark 4.3. The cancellation in the nonlinear terms above is critical to the argument below. We will be able to use the sign of the term and the standard star-shaped geometric condition Assumption 2 on $\Gamma$ to discard the nonlinear boundary contribution from our energy approach.

$$
\begin{aligned}
-2 \int_{0}^{T} & \int_{\Gamma} \partial_{\nu}(\Delta u)(h \cdot \nabla u)-\int_{0}^{T}\left(\gamma-\|\nabla u\|^{2}\right) \int_{\Gamma}(h \cdot \nu)\left|\partial_{\nu} u\right|^{2} \\
= & 2 \int_{0}^{T} \int_{\Gamma} \partial_{\nu}\left(A^{-1} u_{t t}\right)(h \cdot \nabla u)-2 \int_{0}^{T} \int_{\Gamma} \partial_{\nu} D_{L}\left(\left.\Delta u\right|_{\Gamma}\right)(h \cdot \nabla u) \\
& +\gamma \int_{0}^{T} \int_{\Gamma}(h \cdot \nu)\left(\partial_{\nu} u\right)^{2}-\int_{0}^{T}\|\nabla u\|^{2} \int_{\Gamma}(h \cdot \nu)\left(\partial_{\nu} u\right)^{2} \\
& -2 \int_{0}^{T} \int_{\Gamma} \partial_{\nu}\left(A^{-1} p(\mathbf{x})\right)(h \cdot \nabla u) .
\end{aligned}
$$

Now, we estimate the integrals on the RHS of the above inequality, term by term. Using integration by parts in time we note that

$$
\int_{0}^{T} \int_{\Gamma}\left(\partial_{\nu}\left(A^{-1} u_{t t}\right)(h \cdot \nabla u)=\left.\left(\partial_{\nu}\left(A^{-1} u_{t}\right), \partial_{\nu} u\right)_{\Gamma}\right|_{0} ^{T}-\int_{0}^{T} \int_{\Gamma}(h \cdot \nu)\left(\partial_{\nu}\left(A^{-1} u_{t}\right)\left(\partial_{\nu} u_{t}\right) .\right.\right.
$$

From the elliptic regularity theorem [24, Ch. 3]: for any $h \in L^{2}(\Omega)$, we note $\left\|A^{-1} h\right\|_{2} \leq C\|h\|_{0, \Omega}$, and thus we have:

$$
\left\|\partial_{\nu}\left(A^{-1} h\right)\right\|_{0, \Gamma} \leq C\left\|A^{-1} h\right\|_{3 / 2+\epsilon, \Omega} \leq C\left\|A^{-1} h\right\|_{2, \Omega} \leq C\|h\|_{0, \Omega} .
$$

Hence, compactness of the Sobolev embeddings (and Lions' Lemma [20, p.108]), the Hölder-Young 
inequality yields that

$$
\int_{0}^{T} \int_{\Gamma}\left(\partial_{\nu}\left(A^{-1} u_{t t}\right)(h \cdot \nabla u) \leq \epsilon \widehat{E}(T)+C(\epsilon)\|u(t)\|_{0, \Omega}+C \widehat{E}(0)+\epsilon \int_{0}^{T} \widehat{E}(\tau)+C(\epsilon) \int_{0}^{T}\left\|\partial_{\nu} u_{t}\right\|_{0, \Gamma}^{2}\right.
$$

Again, from standard elliptic theory, $\partial_{\nu} D_{L} \in \mathscr{L}\left(L^{2}(\Gamma), H^{-1}(\Gamma)\right)$. Thus we have

$$
\begin{aligned}
\left|\int_{0}^{T} \int_{\Gamma} \partial_{\nu} D_{L}\left(\left.\Delta u\right|_{\Gamma}\right)(h \cdot \nabla u)\right| \leq & \int_{0}^{T}\left\|\partial_{\nu} D_{L}\left(\left.\Delta u\right|_{\Gamma}\right)\right\|_{H^{-1}(\Gamma)}\|h \cdot \nabla u\|_{H^{1}(\Gamma)} \\
\leq & C(h)\left(\left\|D\left(\partial_{\nu} u_{t}\right)\right\|_{L^{2}\left(0, T ; L^{2}(\Gamma)\right)}\right) \times \\
& \left\{\int_{0}^{T}\left\|\frac{\partial^{2} u}{\partial \nu^{2}}\right\|_{L^{2}(\Gamma)}^{2}+\left\|\frac{\partial^{2} u}{\partial \tau \partial \nu}\right\|_{L^{2}(\Gamma)}^{2}\right\}^{1 / 2}
\end{aligned}
$$

and similarly,

$$
\int_{0}^{T} \int_{\Gamma} \partial_{\nu}\left(A^{-1} p(\mathbf{x})\right)(h \cdot \nabla u) \leq \epsilon \int_{0}^{T} \widehat{E}(\tau)+C(p, \epsilon) \cdot T .
$$

Taking into account 4.17 4.19 in 4.16), considering $u=0$ on $\Gamma$, employing Assumption 2, using (4.1), and applying the Hölder-Young we thus arrive at the next preliminary estimate which can be implemented in (4.11):

$$
\begin{aligned}
&-2 \int_{0}^{T} \int_{\Gamma} \partial_{\nu}(\Delta u)(h \cdot \nabla u)-\int_{0}^{T}\left(\gamma-\|\nabla u\|^{2}\right) \int_{\Gamma}(h \cdot \nu)\left(\partial_{\nu} u\right)^{2} \\
& \leq C(h)\left(\left\|D\left(\partial_{\nu} u_{t}\right)\right\|_{L^{2}\left(0, T ; L^{2}(\Gamma)\right)}\right)\left\{\int_{0}^{T}\left\|\frac{\partial^{2} u}{\partial \nu^{2}}\right\|_{L^{2}(\Gamma)}^{2}+\left\|\frac{\partial^{2} u}{\partial \tau \partial \nu}\right\|_{L^{2}(\Gamma)}^{2}\right\}^{1 / 2} \\
&+C(\epsilon) \int_{0}^{T}\left\|\partial_{\nu} u_{t}\right\|_{L^{2}(\Gamma)}^{2}+\epsilon\left\{\widehat{E}(T)+\int_{0}^{T} \widehat{E}(\tau)\right\}+C \widehat{E}(0)+C(p, \epsilon, \gamma, h) \cdot T \\
&+\gamma \int_{0}^{T} \int_{\Gamma}(h \cdot \nu)\left(\partial_{\nu} u\right)^{2} .
\end{aligned}
$$

For the terms in the last line of 4.11) involving the higher order trace term $\partial_{\nu}(h \cdot \nabla u)$, we note the boundary condition $\Delta u=-D\left(\partial_{\nu} u_{t}\right)$ on $\Gamma$, and we have

$$
\begin{aligned}
\int_{0}^{T} & \int_{\Gamma} D\left(\partial_{\nu} u_{t}\right)\left(\partial_{\nu} u\right)+\int_{0}^{T} \int_{\Gamma} 2(\Delta u) \partial_{\nu}(h \cdot \nabla u) \leq \\
& \left\|D\left(\partial_{\nu} u_{t}\right)\right\|_{L^{2}(0, T ; \Gamma)}\left\|\partial_{\nu} u\right\|_{L^{2}(0, T ; \Gamma)}+\left\|D\left(\partial_{\nu} u_{t}\right)\right\|_{L^{2}\left(0, T ; L^{2}(\Gamma)\right)}\left\|\partial_{\nu}(h \cdot \nabla u)\right\|_{L^{2}\left(0, T ; L^{2}(\Gamma)\right)} .
\end{aligned}
$$


We now combine 4.21 and 4.22 in 4.11), and absorb terms to obtain:

$$
\begin{aligned}
\int_{0}^{T} \widehat{E}(\tau) \leq & C(h)\left(\left\|D\left(\partial_{\nu} u_{t}\right)\right\|_{L^{2}\left(0, T ; L^{2}(\Gamma)\right)}\right)\left\{\int_{0}^{T}\left\|\frac{\partial^{2} u}{\partial \nu^{2}}\right\|_{L^{2}(\Gamma)}^{2}+\left\|\frac{\partial^{2} u}{\partial \tau \partial \nu}\right\|_{L^{2}(\Gamma)}^{2}\right\}^{1 / 2} \\
& +C(\epsilon) \int_{0}^{T}\left\|\partial_{\nu} u_{t}\right\|_{L^{2}(\Gamma)}^{2}+\epsilon \widehat{E}(T)+C \widehat{E}(0)+C(p, \epsilon, \gamma, h) T \\
& +C\left\|D\left(\partial_{\nu} u_{t}\right)\right\|_{L^{2}(0, T ; \Gamma)}\left\|\partial_{\nu} u\right\|_{L^{2}(0, T ; \Gamma)}+C\left\|D\left(\partial_{\nu} u_{t}\right)\right\|_{L^{2}\left(0, T ; L^{2}(\Gamma)\right)}\left\|\partial_{\nu}(h \cdot \nabla u)\right\|_{L^{2}\left(0, T ; L^{2}(\Gamma)\right)}
\end{aligned}
$$

Now, considering (4.11) over the interval $(\alpha, T-\alpha)$ instead of $(0, T)$ (hence, performing the calculations above on $(\alpha, T-\alpha)$ ), we can use the decreasing nature (modulo a constant) of the energy functional (1.10) and 4.3) , and then extend the non-critical integrals back on $(0, T)$. We obtain:

$$
\begin{aligned}
\int_{\alpha}^{T-\alpha} \widehat{E}(\tau) \leq & C(h)\left(\left\|D\left(\partial_{\nu} u_{t}\right)\right\|_{L^{2}\left(0, T ; L^{2}(\Gamma)\right)}\right)\left\{\int_{\alpha}^{T-\alpha}\left\|\frac{\partial^{2} u}{\partial \nu^{2}}\right\|_{L^{2}(\Gamma)}^{2}+\left\|\frac{\partial^{2} u}{\partial \tau \partial \nu}\right\|_{L^{2}(\Gamma)}^{2}\right\}^{1 / 2} \\
& +C(\epsilon) \int_{0}^{T}\left\|\partial_{\nu} u_{t}\right\|_{L^{2}(\Gamma)}^{2}+\epsilon \widehat{E}(T-\alpha)+C \widehat{E}(\alpha)+C(p, \epsilon, \gamma, h) \cdot[T-2 \alpha] \\
& +C\left\|D\left(\partial_{\nu} u_{t}\right)\right\|_{L^{2}(0, T ; \Gamma)}\left\|\partial_{\nu} u\right\|_{L^{2}(\alpha, T-\alpha ; \Gamma)} \\
& +C\left\|D\left(\partial_{\nu} u_{t}\right)\right\|_{L^{2}\left(0, T ; L^{2}(\Gamma)\right)}\left\|\partial_{\nu}(h \cdot \nabla u)\right\|_{L^{2}\left(\alpha, T-\alpha ; L^{2}(\Gamma)\right)}
\end{aligned}
$$

The following sharp regularity result for the boundary traces of solutions to the Euler-Bernoulli equation (linear, with given RHS) will be critically used:

Theorem 4.3. ([25, p.281, Theorem 2.4 ]) Let $0<\alpha<T, 0<\delta<1 / 2$, and $s_{0}<1 / 2$ be arbitrary. Then the generalized solutions of (1.1) with $(H D)$ boundary conditions enjoy the bound

$$
\begin{aligned}
{\left[\int_{\alpha}^{T-\alpha} \int_{\Gamma}\left|\partial_{\tau \tau} u\right|^{2}+\left|\partial_{\nu \nu} u\right|^{2}\right.} & \left.+\left|\partial_{\tau \nu} u\right|^{2} d \Gamma d t\right]^{1 / 2} \leq C(T, \alpha, \delta)\left\{\|f(u)\|_{L^{2}\left(0, T ; H^{-s_{0}}(\Omega)\right)}\right. \\
+ & \left.=\|u\|_{L^{2}\left(0, T ; H^{2-\delta}(\Omega)\right)}+\left\|D\left(\partial_{\nu} u_{t}\right)\right\|_{L^{2}\left(0, T ; L^{2}(\Gamma)\right)}+\left\|\partial_{\nu} u_{t}\right\|_{L^{2}\left(0, T ; L^{2}(\Gamma)\right)}\right\}
\end{aligned}
$$

Hereafter, the explicit dependence of the above constants on $\alpha, \delta, \gamma, h$ will be suppressed. For the first term of RHS of above inequality, we make use of 4.25 and recall the nonlinear energy $\widehat{E}$; from 
Young's inequality we obtain:

$$
\begin{aligned}
\left\|D\left(\partial_{\nu} u_{t}\right)\right\|_{L^{2}\left(0, T ; L^{2}(\Gamma)\right)}\left\{\int_{\alpha}^{T-\alpha}\left\|\frac{\partial^{2} u}{\partial \nu^{2}}\right\|_{L^{2}(\Gamma)}^{2}+\left\|\frac{\partial^{2} u}{\partial \tau \partial \nu}\right\|_{L^{2}(\Gamma)}^{2}\right\}^{1 / 2} \\
\leq C+C(T)\left\|D\left(\partial_{\nu} u_{t}\right)\right\|_{L^{2}\left(0, T ; L^{2}(\Gamma)\right)}^{2}\left[\int_{0}^{T}\left\|\left(\gamma-\|\nabla u\|^{2}\right) \Delta u\right\|_{-s_{0}}^{2} d \tau\right] \\
\quad+C(T)\left[\left\|D\left(\partial_{\nu} u_{t}\right)\right\|_{L^{2}\left(0, T ; L^{2}(\Gamma)\right)}^{2}+\left\|\partial_{\nu} u_{t}\right\|_{L^{2}\left(0, T ; L^{2}(\Gamma)\right)}^{2}+\|u\|_{L^{2}\left(0, T ; H^{2-\delta}(\Omega)\right)}^{2}\right] \\
\leq C+C(T)\left\|D\left(\partial_{\nu} u_{t}\right)\right\|_{L^{2}\left(0, T ; L^{2}(\Gamma)\right)}^{2}\left[\int_{0}^{T}(\widehat{E}(\tau))^{2} d \tau\right] \\
\quad+C(T)\left[\left\|D\left(\partial_{\nu} u_{t}\right)\right\|_{L^{2}\left(0, T ; L^{2}(\Gamma)\right)}^{2}+\left\|\partial_{\nu} u_{t}\right\|_{L^{2}\left(0, T ; L^{2}(\Gamma)\right)}^{2}+C\|u\|_{L^{2}\left(0, T ; H^{2-\delta}(\Omega)\right)}^{2}\right] \\
\leq C+\epsilon \int_{\alpha}^{T-\alpha} \widehat{E}(\tau)+C \widehat{E}(\alpha)+C(T, \epsilon)+C(T)\left\|D\left(\partial_{\nu} u_{t}\right)\right\|_{L^{2}\left(0, T ; L^{2}(\Gamma)\right)}^{2}\left[\int_{0}^{T}(\widehat{E}(\tau))^{2} d \tau\right] \\
\quad+C(T)\left[\left\|D\left(\partial_{\nu} u_{t}\right)\right\|_{L^{2}\left(0, T ; L^{2}(\Gamma)\right)}^{2}+\left\|\partial_{\nu} u_{t}\right\|_{L^{2}\left(0, T ; L^{2}(\Gamma)\right)}^{2}\right] .
\end{aligned}
$$

We proceed with estimating the last line of the RHS of 4.24. Using Young's inequality, the trace theorem, and 4.1) we get

$$
\left\|D\left(\partial_{\nu} u_{t}\right)\right\|_{L^{2}(0, T ; \Gamma)}\left\|\partial_{\nu} u\right\|_{L^{2}(\alpha, T-\alpha ; \Gamma)} \leq \epsilon \int_{\alpha}^{T-\alpha} \widehat{E}(\tau)+C \widehat{E}(\alpha)+\int_{0}^{T}\left\|D\left(\partial_{\nu} u_{t}\right)\right\|_{L^{2}(\Gamma)}^{2}+C(\epsilon) \cdot T .
$$

Considering

$$
\partial_{\nu}(h \cdot \nabla u)=\partial_{\nu} u+(h \cdot \nu) \partial_{\nu \nu} u+(h \cdot \tau) \partial_{\nu \tau} u,
$$

and applying similar steps as before, we have:

$$
\begin{aligned}
& \left\|D\left(\partial_{\nu} u_{t}\right)\right\|_{L^{2}\left(0, T ; L^{2}(\Gamma)\right)}\left\|\partial_{\nu}(h \cdot \nabla u)\right\|_{L^{2}\left(\alpha, T-\alpha ; L^{2}(\Gamma)\right)} \leq \\
& \quad \epsilon \int_{\alpha}^{T-\alpha} \widehat{E}(\tau)+C \widehat{E}(\alpha)+C(T)+C(T)\left\|D\left(\partial_{\nu} u_{t}\right)\right\|_{L^{2}\left(0, T ; L^{2}(\Gamma)\right)}^{2}\left[\int_{0}^{T}(\widehat{E}(\tau))^{2} d \tau\right] \\
& \quad+C(T)\left[\left\|D\left(\partial_{\nu} u_{t}\right)\right\|_{L^{2}\left(0, T ; L^{2}(\Gamma)\right)}^{2}+\left\|\partial_{\nu} u_{t}\right\|_{L^{2}\left(0, T ; L^{2}(\Gamma)\right)}^{2}\right] .
\end{aligned}
$$

If we take into account the last two inequalities we have:

$$
\begin{aligned}
\left\|D\left(\partial_{\nu} u_{t}\right)\right\|_{L^{2}(0, T ; \Gamma)} & \left\|\partial_{\nu} u\right\|_{L^{2}(\alpha, T-\alpha ; \Gamma)}+\left\|D\left(\partial_{\nu} u_{t}\right)\right\|_{L^{2}\left(0, T ; L^{2}(\Gamma)\right)}\left\|\partial_{\nu}(h \cdot \nabla u)\right\|_{L^{2}\left(\alpha, T-\alpha ; L^{2}(\Gamma)\right)} \\
\leq & \epsilon \int_{\alpha}^{T-\alpha} \widehat{E}(\tau)+C \widehat{E}(\alpha)+C(T)+C(T)\left\|D\left(\partial_{\nu} u_{t}\right)\right\|_{L^{2}\left(0, T ; L^{2}(\Gamma)\right)}^{2}\left[\int_{0}^{T}(\widehat{E}(\tau))^{2} d \tau\right] \\
& +C(T)\left[\left\|D\left(\partial_{\nu} u_{t}\right)\right\|_{L^{2}\left(0, T ; L^{2}(\Gamma)\right)}^{2}+\left\|\partial_{\nu} u_{t}\right\|_{L^{2}\left(0, T ; L^{2}(\Gamma)\right)}^{2}\right]
\end{aligned}
$$

Finally, we recall that from the energy identity and (1.10), we have for $s \leq t$ :

$$
\begin{aligned}
\widehat{E}(t) & \leq C_{1} \widehat{E}(s)+C_{2} \\
{[t-s] \widehat{E}(t) } & \leq C_{1} \int_{s}^{t} \widehat{E}(\tau) d \tau+C_{2}[t-s]
\end{aligned}
$$


Thus, integrating the energy equality 1.10$)$ on $[\alpha, T-\alpha]$ and employing $(4.3)$, as well as using $(4.26)-$ 4.28 in 4.24, we finally have:

$$
\begin{aligned}
(T-2 \alpha) \widehat{E}(T) \leq & C \widehat{E}(0)+C(T)\left\|D\left(\partial_{\nu} u_{t}\right)\right\|_{L^{2}\left(0, T ; L^{2}(\Gamma)\right)}^{2}\left[\int_{0}^{T}(\widehat{E}(\tau))^{2} d \tau\right] \\
& +C(T)\left[\left\|D\left(\partial_{\nu} u_{t}\right)\right\|_{L^{2}\left(0, T ; L^{2}(\Gamma)\right)}^{2}+\left\|\partial_{\nu} u_{t}\right\|_{L^{2}\left(0, T ; L^{2}(\Gamma)\right)}^{2}\right]+C(T),
\end{aligned}
$$

which is the desired observability estimate, written in terms of the nonlinear, positive energy $\widehat{E}$.

Remark 4.4. Going back to the equation to obtain trace estimates (and making use of the sharp trace result (4.25) above) is the key insight into the present problem. This step connects our observability type estimate to the long-time behavior analysis of [12] by utilizing the fact that the nonlinear energy appears under the time integration against the damping mechanism.

Remark 4.5. We emphasize that at multiple times in the argument above we rely critically on the structure of the Berger nonlinearity - particularly in the ability to drop the term

$$
-\int_{0}^{T}\|\nabla u\|^{2} \int_{\Gamma}(h \cdot \nu)\left|\partial_{\nu} u\right|^{2}
$$

via the geometric condition and in the estimate of higher order trace terms (See Remark 4.4). Since the von Karman nonlinearity does not possess a structure which accommodates the trace estimates needed above, we cannot expect a straightforward approach to yield similar results there. Thus, for the Berger nonlinearity, in addition to the existence of a compact global attractor, we also have an explicit estimate on the absorbing set (not available when the attractor is obtained - for gradient systems - indirectly through the asymptotic smoothness property).

\section{Completion of the Proof of Theorem 4.1}

Now, let us denote

$$
D_{0}^{T}=\int_{0}^{T} \int_{\Gamma} D\left(\partial_{\nu} u_{t}\right)\left(\partial_{\nu} u_{t}\right)
$$

Since $D(0)=0$, we have for $s \geq 2$ that

$$
\begin{aligned}
D(s) & =\int_{0}^{s} D^{\prime}(\tau) d \tau=s \int_{0}^{1} D^{\prime}(\tau s) d \tau \geq \operatorname{sinf}_{s \geq 2} \int_{0}^{1} D^{\prime}(\tau s) d \tau \\
& \geq \operatorname{sinf}_{s \geq 2} \int_{1 / 2}^{1} D^{\prime}(\tau s) d \tau \geq \frac{s}{2} \inf _{s \geq 2} \inf _{1 / 2 \leq \tau \leq 1} D^{\prime}(\tau s)=\frac{s}{2} \inf _{\xi \geq 1} D^{\prime}(\xi) \geq \frac{s}{2} m .
\end{aligned}
$$

It can be shown in an analogous way that for $s \leq-2$ we have $D(s) \leq \frac{s}{2} m$. Additionally, with a given $\epsilon>0$ we have for $s \in(\epsilon, 2)$ :

$$
D(s)=\int_{0}^{s} D^{\prime}(\tau) d \tau \geq \int_{0}^{\epsilon} D^{\prime}(\tau) d \tau \geq m_{\epsilon} \frac{s}{2}
$$


with a same inequality for $s \in(-2,-\epsilon)$. Hence the above relations give that:

$$
D(s) s \geq \frac{s^{2}}{2} m \quad \text { for }|s| \geq 2 \quad \text { and } \quad D(s) s \geq m_{\epsilon} \frac{s^{2}}{2} \quad \text { for }|s| \geq \epsilon .
$$

Similarly, one can show for $s \geq 0$ that

$$
D(s) \leq s \cdot \max \left\{\sup _{0 \leq|\xi| \leq 1} D^{\prime}(\xi), M\right\} .
$$

Hence we obtain

$$
(D(s))^{2} \leq D(s) s \max \left\{\sup _{0 \leq|\xi| \leq 1} D^{\prime}(\xi), M\right\} .
$$

Now, considering the above relations we obtain that

$$
\left[\left\|D\left(\partial_{\nu} u_{t}\right)\right\|_{L^{2}\left(0, T ; L^{2}(\Gamma)\right)}^{2}+\left\|\partial_{\nu} u_{t}\right\|_{L^{2}\left(0, T ; L^{2}(\Gamma)\right)}^{2}\right] \leq C(T, \Gamma)+\max \left\{\frac{2}{m}, \sup _{0 \leq|\xi| \leq 1} D^{\prime}(\xi), M\right\} D_{0}^{T}
$$

If we take into account 4.32 in our observability estimate 4.5 we have

$$
(T-2 \alpha) \widehat{E}(\tau) d \tau \leq C \widehat{E}(0)+C(T) D_{0}^{T}+C(T) D_{0}^{T} \int_{0}^{T}(\widehat{E}(\tau))^{2}+K(T)
$$

where $K(T)$ does not depend on $\widehat{E}(0)$. Since

$$
\widehat{E}(0) \leq D_{0}^{T}+C \widehat{E}(T)+C(p, \gamma),
$$

we have

$$
(T-2 \alpha-C) \widehat{E}(T) \leq C(T) D_{0}^{T}\left[1+\int_{0}^{T}(\widehat{E}(\tau))^{2} d \tau\right]+K(T)
$$

Now, if we rewrite the last inequality in terms of the full nonlinear enery $\mathscr{E}(T)$, use the relation (4.3) between $\mathscr{E}$ and $\widehat{E}$, and employ the notation $\mathscr{E}_{M}(T) \equiv \mathscr{E}(T)+M$, where $M$ is the constant coming from 4.4, we obtain:

$$
(T-2 \alpha-C) \mathscr{E}_{M}(T) \leq C(T) D_{0}^{T}\left[1+\int_{0}^{T} \mathscr{E}_{M}^{2}(\tau) d \tau\right]+K(T)
$$

Using the fact that $\mathscr{E}_{M}(T) \leq \mathscr{E}_{M}(0)$, and $D_{0}^{T}=\mathscr{E}_{M}(0)-\mathscr{E}_{M}(T)$, we have

$$
(T-2 \alpha-C) \mathscr{E}_{M}(T) \leq C(T)\left[\mathscr{E}_{M}(0)-\mathscr{E}_{M}(T)\right]\left[1+T \mathscr{E}_{M}^{2}(0)\right]+K(T),
$$


from which we obtain

$$
\left[(T-2 \alpha-C)+C(T)\left[1+T \mathscr{E}_{M}^{2}(0)\right]\right] \mathscr{E}_{M}(T) \leq C(T)\left[1+T \mathscr{E}_{M}^{2}(0)\right] \mathscr{E}_{M}(0)+K(T)
$$

Then, assuming $T$ large enough and recalling 4.3 , we have

$$
\mathscr{E}_{M}(T) \leq \frac{C(\widehat{E}(0), T)}{T+C(\widehat{E}(0), T)} \mathscr{E}_{M}(0)+\frac{K(T)}{T+C(\widehat{E}(0), T)}
$$

Denoting $\eta(\widehat{E}(0), T)=\frac{C(\widehat{E}(0), T)}{T+C(\widehat{E}(0), T)}<1$, we rewrite the last inequality as

$$
\mathscr{E}_{M}(T) \leq \eta(\widehat{E}(0), T) \mathscr{E}_{M}(0)+\frac{K(T)}{T+C(\widehat{E}(0), T)} .
$$

Now, we can reiterate the same estimate on each subinterval $(m T,(m+1) T)$ via the semigroup property. We note that the constants $C(\widehat{E}(0), T)$ and $K(T)$ will be same at each step. Then we obtain

$$
\begin{aligned}
\mathscr{E}_{M}((m+1) T) \leq & \frac{C(\widehat{E}(0), T)}{T+C(\widehat{E}(0), T)} \mathscr{E}_{M}(m T)+\frac{K(T)}{T+C(\widehat{E}(0), T)} \\
& \leq \eta(\widehat{E}(0), T)^{m} \mathscr{E}_{M}(0)+\sum_{i=0}^{m} \eta(\widehat{E}(0), T)^{i} \frac{K(T)}{T+C(\widehat{E}(0), T)} \\
& \leq \eta(\widehat{E}(0), T)^{m} \mathscr{E}_{M}(0)+\left[\frac{1}{1-\eta(\widehat{E}(0), T)}\right] \frac{K(T)}{T+C(\widehat{E}(0), T)} \\
& \equiv \eta(\widehat{E}(0), T)^{m} \mathscr{E}(0)+\bar{K}(T)
\end{aligned}
$$

The monotonicity and continuity of the energy function, and the fact that $\eta(\widehat{E}(0), T)<1$, gives

$$
\mathscr{E}_{M}(t) \leq \bar{K}(T)+1, \quad \text { for all } \quad t>t_{0}(\widehat{E}(0))
$$

which together with 4.3 completes the proof of Theorem 4.1

\subsubsection{Asymptotic Smoothness - Completion of the Proof of Theorem 1.3}

In this section, as the second step of the proof of Theorem 1.3 we show the asymptotic smoothness property of the dynamical system generated by (1.1) with (HD) boundary conditions in the following theorem:

Theorem 4.4. Let Assumptions 1 and 2 be in force. The dynamical system $\left(S_{H}(\cdot), \mathscr{H}\right)$ generated by generalized solutions to (1.1) taken with (HD) boundary conditions is asymptotically smooth.

We note that after Theorem 4.4 is proved, our main theorem on the existence of a global attractor for generalized solutions to (1.1) taken with (HD) conditions (Theorem 1.3) will be proved, via Theorem 6.1

Proof of Theorem 4.4. The proof rests on the application of Theorem 6.3 Hence we are interested in the difference of two solutions $z=u-w$, where $U(t)=\left(u(t), u_{t}(t)\right)=S_{H}(t) y_{1}$ and $W(t)=$ 
$\left(w(t), w_{t}(t)\right)=S_{H}(t) y_{2}$ solve (1.1) corresponding to initial conditions $y_{1}$ and $y_{2}$ (respectively), taken from an invariant, bounded set. Then, $z$ will solve the following problem:

$$
\begin{aligned}
& z_{t t}+\Delta^{2} z+\mathcal{F}(z)=0 \quad \text { in }(0, T) \times \Omega ; \quad z=0, \quad \Delta z=-\left[D\left(\partial_{\nu} u_{t}\right)-D\left(\partial_{\nu} w_{t}\right)\right] \text { on } \Gamma, \\
& z(0)=u_{0}-w_{0}, \quad z_{t}(0)=u_{1}-w_{1},
\end{aligned}
$$

where $\mathcal{F}(z) \equiv f_{B}(u)-f_{B}(w)$. We denote the energy for the difference: $\quad E_{z}(t)=\frac{1}{2}\left\{\|\Delta z\|^{2}+\left\|z_{t}(t)\right\|^{2}\right\}$. The energy identity $(1.10)$, the relation 4.3 between the energies, and the bound on lower frequencies 4.1), give that there exists an $R_{*}$ such that the set

$$
\mathscr{W}_{R} \equiv\left\{\left(u_{0}, u_{1}\right) \in \mathscr{H}: \mathscr{E}\left(u_{0}, u_{1}\right) \leq R\right\}
$$

is a non-empty bounded set in $\mathscr{H}$ for all $R \geq R_{*}$. Moreover, any bounded set $\mathscr{B} \subset \mathscr{H}$ is contained in $\mathscr{W}_{R}$ for some $R$, and the set $\mathscr{W}_{R}$ is invariant with respect to $S_{H}(t)$. Then, we consider the restriction of the dynamical system $\left(S_{H}(\cdot), \mathscr{H}\right)$ to $\left(S_{H}(\cdot), \mathscr{W}_{R}\right)$ in showing the asymptotic smoothness property, and thus we consider the solutions $u, w$ satisfying

$$
\|u(t)\|_{2}+\left\|u_{t}(t)\right\|_{0}+\|w(t)\|_{2}+\left\|w_{t}(t)\right\|_{0} \leq C(R), \quad t>0 .
$$

The main ingredient will be the following estimate on the difference of the solutions:

Lemma 4.5. Let $T>0$ and $\beta \in C^{2}(\mathbb{R})$ be a given function satisfying (i) $\operatorname{supp}(\beta) \subset[\alpha, T-\alpha]$ (with $\alpha<T / 2$ ); (ii) $0 \leq \beta \leq 1$ and $\beta \equiv 1$ on $[\alpha, T-\alpha]$. Then, any solution $z$ to equation (4.33) satisfies

$$
\begin{aligned}
\int_{0}^{T} E_{z}(t) \beta(t) d t & \leq C_{1} \int_{0}^{T} E_{z}(t)\left|\beta^{\prime}(t)\right| d t+C_{2}(T)\left\{\int_{0}^{T} \int_{\Gamma}\left[D\left(\partial_{\nu} u_{t}\right)-D\left(\partial_{\nu} w_{t}\right)\right] \partial_{\nu} z_{t}+\left|\partial_{\nu} z_{t}\right|^{2} d \Gamma\right\} \\
& + \text { l.o.t. }(z),
\end{aligned}
$$

where

$$
\text { l.o.t. }(z) \equiv C(T, R)\left[\sup _{[0, T]}\|z(t)\|_{2-\eta}^{2}+\int_{0}^{T}\left\|z_{t}(\tau)\right\|_{-\eta}^{2} d \tau\right], \quad 0<\eta<1 / 2 .
$$

Proof. The proof of this lemma follows [14, Section 10.5] exactly, with respect to the linear portion of the dynamics and the nonlinear damping: the application of the flux multiplier $h \cdot \nabla(\beta z)$, where $h=\mathbf{x}-\mathbf{x}_{0}, \mathbf{x}_{0} \in \mathbb{R}^{2}$, the use of sharp trace results proved in [25] (as in the previous section), the local Lipschitz property of $f$, and the employment of Assumption 1. This yields the above estimate (4.34).

Now, by the energy relation,

$$
E_{z}(T)+\int_{t}^{T} \int_{\Gamma}\left[D\left(\partial_{\nu} u_{t}\right)-D\left(\partial_{\nu} w_{t}\right)\right]\left(\partial_{\nu} z_{t}\right)+\int_{t}^{T}\left(\mathcal{F}(z), z_{t}\right)=E_{z}(t), \quad T \geq t,
$$

we have: 
(i)

$$
\begin{aligned}
& \int_{0}^{T} E_{z}(t)\left(1-\beta(t)+\left|\beta^{\prime}(t)\right|\right) d t \leq \\
& \quad \int_{0}^{T}\left[1-\beta(t)+\left|\beta^{\prime}(t)\right|\right] d t\left[E_{z}(T)+\int_{0}^{T} \int_{\Gamma}\left[D\left(\partial_{\nu} u_{t}\right)-D\left(\partial_{\nu} w_{t}\right)\right]\left(\partial_{\nu} z_{t}\right)\right]+\mathcal{F}_{*}(z),
\end{aligned}
$$

where

$$
\mathcal{F}_{*}(z) \equiv \int_{0}^{T}\left(1-\beta(t)-\left|\beta^{\prime}(t)\right|\right) \int_{t}^{T}\left(\mathcal{F}(z), z_{t}\right) d \tau d t
$$

(ii)

$$
T E_{z}(T) \leq \int_{0}^{T} E_{z}(t) d t+\mathcal{F}_{* *}(z)
$$

where

$$
\mathcal{F}_{* *}(z) \equiv-\int_{0}^{T} \int_{t}^{T}\left(\mathcal{F}(z), z_{t}\right) d \tau d t
$$

Combining (i) and (ii), we obtain that there exist $T_{0}>0$, and constants $C_{1}(T)$ and $C_{2}(R, T)$, such that

$$
\begin{aligned}
T E_{z}(T)+\int_{0}^{T} E_{z}(t) d t \leq & C_{1}(T)\left[\int_{0}^{T} \int_{\Gamma}\left[D\left(\partial_{\nu} u_{t}\right)-D\left(\partial_{\nu} w_{t}\right)\right]\left(\partial_{\nu} z_{t}\right) d \Gamma d t+\int_{0}^{T} \int_{\Gamma}\left|\partial_{\nu} z_{t}\right|^{2} d \Gamma d t\right] \\
& +\mathcal{F}_{*}(z)+\mathcal{F}_{* *}(z)+C_{2}(R, T) \text { l.o.t.(z), } \quad \forall T \geq T_{0} .
\end{aligned}
$$

Using the assumption on the structure of the damping (Assumption 1), we have

$$
\int_{0}^{T} \int_{\Gamma}\left|\partial_{\nu} z_{t}\right|^{2} \leq \epsilon+C(\epsilon) \int_{0}^{T} \int_{\Gamma}\left[D\left(\partial_{\nu} u_{t}\right)-D\left(\partial_{\nu} w_{t}\right)\right]\left(\partial_{\nu} z_{t}\right), \quad \forall \epsilon>0 .
$$

On the other hand, it follows immediately from the energy relation 4.35 that

$$
\int_{0}^{T} \int_{\Gamma}\left[D\left(\partial_{\nu} u_{t}\right)-D\left(\partial_{\nu} w_{t}\right)\right]\left(\partial_{\nu} z_{t}\right) \leq E_{z}(0)-E_{z}(T)+\left|\int_{0}^{T}\left(\mathcal{F}(z), z_{t}\right)\right| .
$$

Now, taking into account the last two inequalities in 4.36, we obtain

$$
\begin{aligned}
T E_{z}(T)+\int_{0}^{T} E_{z}(t) d t \leq & \epsilon+C_{1}(\epsilon, T)\left[E_{z}(0)-E_{z}(T)\right]+C_{2}(\epsilon, T)\left|\int_{0}^{T}\left(\mathcal{F}(z), z_{t}\right)\right| \\
& +\mathcal{F}_{*}(z)+\mathcal{F}_{* *}(z)+C_{3}(\epsilon, T, R) \text { l.o.t. }(z)
\end{aligned}
$$

for any $\epsilon>0$.

In order to apply Theorem 6.3, we need to simplify the nonlinear terms above. For this, we demonstrate a decomposition of Berger nonlinearity for the difference of two solutions.

Lemma 4.6. Let $z=u-w$, and let $f_{B}(u)=\left(\gamma-\|\nabla u\|^{2}\right) \Delta u$, and $\mathcal{F}(z)=f_{B}(u)-f_{B}(w)$. Also 
assume $u, w \in C\left([t, T] ;\left(H^{2} \cap H_{0}^{1}\right)(\Omega)\right) \cap C^{1}\left([t, T] ; L^{2}(\Omega)\right)$. Then

$$
\begin{aligned}
\int_{t}^{T}\left(\mathcal{F}(z), z_{t}\right)_{\Omega} d \tau= & {\left[(\mathcal{F}(z), z)_{\Omega}+\frac{\gamma}{2}\|\nabla z\|^{2}-\frac{1}{2}\|\nabla u\|^{2}\|\nabla z\|^{2}-\left(\|\nabla u\|^{2}-\|\nabla w\|^{2}\right)(\Delta w, z)_{\Omega}\right]_{t}^{T} } \\
& -\int_{t}^{T}\left(\|\nabla u\|^{2}-\|\nabla w\|^{2}\right)\left(\Delta w, z_{t}\right)_{\Omega} d \tau+\int_{t}^{T}\left(\Delta u, u_{t}\right)_{\Omega}\|\nabla z\|^{2} d \tau
\end{aligned}
$$

Proof. Letting $z=u-w$, we note

$$
\begin{aligned}
\int_{t}^{T}\left(\mathcal{F}(z), z_{t}\right) d \tau & =-\int_{t}^{T}\left(\left[\|\nabla u\|^{2} \Delta u-\|\nabla w\|^{2} \Delta w\right], z_{t}\right) d \tau+\gamma \int_{t}^{T}\left(\Delta z, z_{t}\right) d \tau \\
& =\int_{t}^{T}\left(\left[\|\nabla u\|^{2} \Delta u\right]^{\prime}-\left[\|\nabla w\|^{2} \Delta w\right]^{\prime}, z\right) d \tau+\left[(\mathcal{F}(z), z)+\frac{\gamma}{2}\|\nabla z\|^{2}\right]_{t}^{T}
\end{aligned}
$$

where we have integrated by parts in time, integrated by parts in space on the term with $\gamma$, and recognized the total derivative term $\left(\nabla z, \nabla z_{t}\right)$. We now focus on the nonlinear term and observe

$$
\begin{aligned}
\int_{t}^{T}\left(\left[\|\nabla u\|^{2} \Delta u\right]^{\prime}-\left[\|\nabla w\|^{2} \Delta w\right]^{\prime}, z\right) d \tau= & \int_{t}^{T}\left(\left(\|\nabla u\|^{2}\right)^{\prime} \Delta u-\left(\|\nabla w\|^{2}\right)^{\prime} \Delta w, z\right) d \tau \\
& +\int_{t}^{T}\left(\|\nabla u\|^{2} \Delta u_{t}-\|\nabla w\|^{2} \Delta w^{\prime}, z\right) d \tau
\end{aligned}
$$

If we add and subtract the terms which are mixed in $u$ and $w$ we have

$$
\begin{aligned}
\int_{t}^{T}\left(\left[\|\nabla u\|^{2} \Delta u\right]^{\prime}\right. & \left.-\left[\|\nabla w\|^{2} \Delta w\right]^{\prime}, z\right) d \tau= \\
& \int_{t}^{T}\left[\left(\|\nabla u\|^{2}\right)^{\prime}(\Delta z, z)+\left[\left(\|\nabla u\|^{2}\right)^{\prime}-\left(\|\nabla w\|^{2}\right)^{\prime}\right](\Delta w, z)\right] d \tau \\
& +\int_{t}^{T}\left[\|\nabla u\|^{2}\left(\Delta z_{t}, z\right)+\left[\|\nabla u\|^{2}-\|\nabla w\|^{2}\right]\left(\Delta w_{t}, z\right)\right] d \tau .
\end{aligned}
$$

Now, recognizing a total derivative and adjusting we arrive at

$$
\begin{aligned}
\int_{t}^{T}\left(\left[\|\nabla u\|^{2} \Delta u\right]^{\prime}\right. & \left.-\left[\|\nabla w\|^{2} \Delta w\right]^{\prime}, z\right) d \tau \\
= & -\int_{t}^{T}\left[\frac{d}{d \tau}\left[\|\nabla u\|^{2}\|\nabla z\|^{2}\right]-\frac{1}{2}\|\nabla u\|^{2}\left(\|\nabla z\|^{2}\right)^{\prime}\right] d \tau \\
& -\int_{t}^{T}\left[\frac{d}{d \tau}\left[\left(\|\nabla u\|^{2}-\|\nabla w\|^{2}\right)(\Delta w, z)\right]+\left(\|\nabla u\|^{2}-\|\nabla w\|^{2}\right)\left(\Delta w, z_{t}\right)\right] d \tau \\
= & -\left[\frac{1}{2}\|\nabla u\|^{2}\|\nabla z\|^{2}+\left(\|\nabla u\|^{2}-\|\nabla w\|^{2}\right)(\Delta w, z)\right]_{t}^{T} \\
& -\int_{t}^{T}\left(\|\nabla u\|^{2}-\|\nabla w\|^{2}\right)\left(\Delta w, z_{t}\right) d \tau+\int_{t}^{T}\left(\Delta u, u_{t}\right)\|\nabla z\|^{2} d \tau
\end{aligned}
$$

which is the desired decomposition. 
At this point, noting that

$$
\|u(t)\|_{2}+\left\|u_{t}(t)\right\|_{0}+\|w(t)\|_{2}+\left\|w_{t}(t)\right\|_{0} \leq C(R), \quad t>0,
$$

using triangle inequality

$$
\begin{aligned}
\left|\|\nabla u\|^{2}-\|\nabla w\|^{2}\right| & =|\|\nabla u\|-\|\nabla w\||(|| \nabla u\|+\| \nabla w \|) \\
& \leq\|\nabla u-\nabla w\|(\|\nabla u\|+\|\nabla w\|) \\
& \leq C(R)\|z\|_{1}
\end{aligned}
$$

and taking into account the last two inequalities in Lemma 4.6 we obtain the key inequality:

$$
\left|\int_{t}^{T}\left(\mathcal{F}(z), z_{t}\right) d \tau\right| \leq \epsilon \int_{t}^{T} E_{z}(\tau) d \tau+C(\epsilon, R, T) \sup _{[t, T]}\|z\|_{2-\eta}^{2}, \quad \eta>0 .
$$

From this, the analogous bounds on $\mathcal{F}_{*}$ and $\mathcal{F}_{* *}$ follow.

\section{Completion of the Proof of Theorem 4.4-Asymptotic Smoothness}

We are now in a position to finish the proof of Theorem 4.4. Firstly, implementing 4.38 in (4.37) and rescaling $\epsilon$, we see that

$$
E_{z}(T) \leq \epsilon+C_{1}(\epsilon, T)\left[E_{z}(0)-E_{z}(T)\right]+\text { l.o.t. }(z) .
$$

In order to invoke Theorem 6.3 we need only to construct a functional $\Psi_{\epsilon, R, T}$ satisfying the compensated compactness condition [14, 21], i.e.,

$$
\liminf _{k \rightarrow \infty} \liminf _{n \rightarrow \infty} \Psi_{\epsilon, R, T}\left(y_{n}, y_{k}\right)=0
$$

for every sequence $\left\{y_{n}\right\}$ from $\mathscr{B} \subset \mathscr{H}$. For this, we use a standard stabilization type argument via the semigroup property of the dynamics on the interval $((m-1) T, m T)$. This yields

$$
E_{z}(m T) \leq \frac{\epsilon}{1+C(\epsilon, R, T)}+\gamma_{\epsilon} E_{z}((m-1) T)+\text { l.o.t. }^{m}(z),
$$

where

$$
\gamma_{\epsilon}=\frac{C(\epsilon, R, T)}{1+C(\epsilon, R, T)}<1
$$

and

$$
\text { l.o.t. }^{m}(z)=C(\epsilon, R, T)\left[\sup _{[(m-1) T, m T]}\|z(\tau)\|_{2-\eta}^{2}+\int_{(m-1) T}^{m T}\left\|z_{t}(\tau)\right\|_{-\eta}^{2} d \tau\right] .
$$

After iteration, we observe that

$$
E_{z}(m T) \leq \epsilon+\gamma_{\epsilon}^{m} E_{z}(0)+\sum_{j=0}^{m-1} \gamma_{\epsilon}^{j} \text { l.o.t. }{ }^{m-j}(z) .
$$


For any $\epsilon_{0}$, we may select $\epsilon>0$, then $m$ large enough so that, with $R$ and $T$ fixed, we have

$$
E_{z}(m T) \leq \epsilon_{0}+\sum_{j=0}^{m-1} \gamma_{\epsilon}^{j} \text { l.o.t. }{ }^{m-j}(z)
$$

Now, for $y_{1}=U(0)$ and $y_{2}=W(0)$, setting

$$
\Psi_{\epsilon_{0}, \mathscr{B}, m T}\left(y_{1}, y_{2}\right) \equiv \sum_{j=0}^{m-1} \gamma_{\epsilon}^{j} \text { l.o.t. }^{m-j}(z)
$$

the functional $\Psi_{\epsilon_{0}, \mathscr{B}, m T}$ - being compact with respect to the energy space $\mathscr{H}$ (via the Sobolev embeddings) satisfies the requisite condition of the functional $\Psi$ in Theorem 6.3. This concludes the proof of asymptotic smoothness of the dynamical system $\left(S_{H}(\cdot), \mathscr{W}_{R}\right)$ for any $R>0$, according to Theorem 6.3

As a result, by Theorem 4.1 and Theorem 4.4 we obtain the existence of global attractor which finishes the proof of Theorem 1.3

\section{Acknowledgements}

The authors would like to thank Professors Azer Khanmamedov and Irena Lasiecka for their (respective) very helpful advice and suggestions. The authors also thank the referee for valuable suggestions which improved this treatment.

J.T. Webster was partially supported by NSF-DMS-1504697 in performing this research.

\section{Appendix}

\subsection{Long-time Behavior of Dynamical Systems}

In the context of this paper we will use a few keys theorems (which we now formally state) to prove the existence of the attractor and determine its properties, as well as provide some context for other results mentioned in the discussions above. For general dynamical systems references, see [4, 27, 30, 14 ] (and refences therein). For proofs pertinent to what is presented here, and more references, see [14].

Let $\left(\mathcal{H}, S_{t}\right)$ be a dynamical system on a complete metric space $\mathcal{H}$ with $\mathscr{N} \equiv\left\{x \in \mathcal{H}: S_{t} x=\right.$ $x$ for all $t \geq 0\}$ the set of its stationary points. $\left(\mathcal{H}, S_{t}\right)$ is said to be dissipative iff it possesses a bounded absorbing set $\mathcal{B}$. This is to say that for any bounded set $B$, there is a time $t_{B}$ so that $S_{t_{B}}(B) \subset \mathcal{B}$. We say that a dynamical system is asymptotically compact if there exists a compact set $K$ which is uniformly attracting: for any bounded set $D \subset \mathcal{H}$ we have that $\lim _{t \rightarrow+\infty} d_{\mathcal{H}}\left\{S_{t} D \mid K\right\}=0$ in the sense of the Hausdorff semidistance. $\left(\mathcal{H}, S_{t}\right)$ is said to be asymptotically smooth if for any bounded, forward invariant $(t>0)$ set $D$ there exists a compact set $K \subset \bar{D}$ which is uniformly attracting (as in the previous definition). Global attractor $\mathbf{A}$ is a closed, bounded set in $\mathcal{H}$ which is invariant (i.e. $S_{t} \mathbf{A}=\mathbf{A}$ for all $t>0$ ) and uniformly attracting.

The following if and only if characterization of global attractors is well-known [4, 14] 
Theorem 6.1. Let $\left(\mathcal{H}, S_{t}\right)$ be a dissipative dynamical system in a complete metric space $\mathcal{H}$. Then $\left(\mathcal{H}, S_{t}\right)$ possesses a compact global attractor $\mathbf{A}$ if and only if $\left(\mathcal{H}, S_{t}\right)$ is asymptotically smooth.

An asymptotically smooth dynamical system for which there is a Lyapunov function $\Phi(x)$ that is bounded from above on any bounded set can be thought of as one which possesses local attractors. To see this stated precisely see [13] page 33. Such a result provides an existence of local attractors, i.e., and attractor for any bounded set of initial data. However, these sets need not be uniformly bounded with respect to the size of the set of initial data. The latter can be guaranteed by the existence of a uniform absorbing set. However, establishing this existence of an absorbing set may be technically demanding. In some instances, there is a way of circumventing this difficulty which takes advantage of the "good" structure of a Lyapunov function.

A strict Lyapunov function for $\left(\mathcal{H}, S_{t}\right)$ is a functional $\Phi$ on $\mathcal{H}$ such that (i) the map $t \rightarrow \Phi\left(S_{t} x\right)$ is nonincreasing for all $x \in \mathcal{H}$, and (ii) $\Phi\left(S_{t} x\right)=\Phi(x)$ for all $t>0$ and $x \in \mathcal{H}$ implies that $x$ is a stationary point of $\left(\mathcal{H}, S_{t}\right)$. If the dynamical system has a strict Lyapunov function defined on the entire phase space, then we say that $\left(\mathcal{H}, S_{t}\right)$ is gradient.

We can address attractors for gradient systems and characterize the attracting set. The following result follows from Theorem 2.28 and Corollary 2.29 in [13].

Theorem 6.2. Suppose that $\left(\mathcal{H}, S_{t}\right)$ is a gradient, asymptotically smooth dynamical system. Suppose its Lyapunov function $\Phi(x)$ is bounded from above on any bounded subset of $\mathcal{H}$ and the set $\Phi_{R} \equiv\{x \in$ $\mathcal{H}: \Phi(x) \leq R\}$ is bounded for every $R$. If the set of stationary points for $\left(\mathcal{H}, S_{t}\right)$ is bounded, then $\left(\mathcal{H}, S_{t}\right)$ possesses a compact global attractor $\mathbf{A}$ which coincides with the unstable manifold, i.e.

$\mathbf{A}=\mathscr{M}^{u}(\mathscr{N}) \equiv\left\{x \in \mathcal{H}: \exists U(t) \in \mathcal{H}, \forall t \in \mathbb{R}\right.$ such that $U(0)=x$ and $\left.\lim _{t \rightarrow-\infty} d_{\mathcal{H}}(U(t) \mid \mathscr{N})=0\right\}$

Secondly, we state a useful criterion (first appearing 21 and stated in the present version in [14]) which reduces asymptotic smoothness to finding a suitable functional on the state space with a compensated compactness condition:

Theorem 6.3. ([13]-Proposition 2.10) Let $\left(\mathcal{H}, S_{t}\right)$ be a dynamical system, $\mathcal{H}$ Banach with norm $\|\cdot\|$. Assume that for any bounded positively invariant set $B \subset \mathcal{H}$ and for all $\epsilon>0$ there exists a $T \equiv T_{\epsilon, B}$ such that

$$
\left\|S_{T} x_{1}-S_{T} x_{2}\right\|_{\mathcal{H}} \leq \epsilon+\Psi_{\epsilon, B, T}\left(x_{1}, x_{2}\right), \quad x_{i} \in B
$$

with $\Psi$ a functional defined on $B \times B$ depending on $\epsilon, T$, and $B$ such that

$$
\liminf _{m} \liminf _{n} \Psi_{\epsilon, T, B}\left(x_{m}, x_{n}\right)=0
$$

for every sequence $\left\{x_{n}\right\} \subset B$. Then $\left(\mathcal{H}, S_{t}\right)$ is an asymptotically smooth dynamical system.

\section{References}

[1] G. Avalos, P.G. Geredeli, and J.T. Webster, Finite dimensional smooth attractors for the Berger plate with dissipation acting on a portion of the boundary, preprint. 
[2] G. Avalos and I. Lasiecka, Exponential Stability of a Thermoelastic System Without Mechanical Dissipation, Rend. Istit. Mat. Univ. Trieste, 28 (1997), pp. 1-28.

[3] G. Avalos and I. Lasiecka, Boundary Controllability of Thermoelastic Plates via the Free Boundary Conditions, SIAM J. Control. Optim., 38 (2000), pp. 337-383.

[4] A. Babin and M. Vishik, Attractors of Evolution Equations, North-Holland, Amsterdam, 1992.

[5] B. Banerjee and S. Datta, A new approach to an analysis of large deflections of thin elastic plates, J. Non-Lin. Mech., 16 (1981), 1, pp. 47-52.

[6] H.M. Berger, A new approach to the analysis of large deflections of plates, J. Appl. Mech., 22 (1955), pp. 465-472.

[7] L. Bociu and D. Toundykov, Attractors for non-dissipative irrotational von Karman plates with boundary damping, JDE, 253 (2012), 12, pp. 3568-3609.

[8] V.V. Bolotin, Nonconservative Problems of Elastic Stability, Pergamon Press, Oxford, 1963.

[9] S.C. Brenner and R. Scott, The mathematical theory of finite element methods, v. 15, Springer Science \& Business Media, 2008.

[10] I. Chueshov, Introduction to the Theory of Infinite Dimensional Dissipative Systems, Acta, Kharkov, 1999, in Russian; English translation: Acta, Kharkov, 2002; see also http://www.emis.de/monographs/Chueshov/

[11] I. Chueshov, Introduction to the Theory of Infinite-Dimensional Dissipative Systems, Acta, Kharkov, 1999 (in Russian); English translation: Acta, Kharkov, 2002; http://www.emis.de/monographs/Chueshov/

[12] I. Chueshov, M. Eller, and I. Lasiecka, Finite dimensionality of the attractor for a semilinear wave equation with nonlinear boundary dissipation, Comm. PDE 29 (2004), pp. 1847-1976.

[13] I. Chueshov and I. Lasiecka, Long-time behavior of second-order evolutions with nonlinear damping, Memoires of AMS, v. 195, 2008.

[14] I. Chueshov and I. Lasiecka, Von Karman Evolution Equations, Springer-Verlag, 2010

[15] P. Ciarlet and P. Rabier, Les Equations de Von Karman, Springer, 1980.

[16] A. Eden and A.J. Milani, Exponential attractors for extensible beam equations, Nonlinearity 6 (1993), pp. 457-479.

[17] P.G. Geredeli, I. Lasiecka, J.T. Webster, Smooth attractors of finite dimension for von Karman evolutions with nonlinear frictional damping localized in a boundary layer, J. Diff. Eqs. 254 (2013), pp. 1193-1229.

[18] P.G. Geredeli, J.T. Webster, Decay rates to eqilibrium for nonlinear plate equations with geometrically constrained, degenerate dissipation, Appl. Math. and Optim. 68 (2013), pp. 361-390. Corrigendum, Appl. Math. and Optim. 70 (2014), pp. 565-566. 
[19] G. Ji and I. Lasiecka, Nonlinear Boundary Feedback Stabilization for a Semilinear Kirchhoff Plate with Dissipation Acting Only via Moments-Limiting Behavior, JMAA 229 (1999), pp. 452-479.

[20] S. Kesavan, Topics in Functional Analysis and Applications, John Wiley and Sons, India, 1989.

[21] A.Kh. Khanmamedov, Global attractors for von Karman equations with non-linear dissipation, J. Math. Anal. Appl 318 (2006), pp. 92-101.

[22] H. Koch and I. Lasiecka, Hadamard well-posedness of weak solutions in nonlinear dynamic elasticity - full von Karman systems. Evolution Equations, Semigroup and Functional Analysis, 50 (2002), pp. 197-212.

[23] J. Lagnese, Boundary Stabilization of Thin Plates, SIAM, 1989.

[24] I. Lasiecka and R. Triggiani, Control Theory for Partial Differential Equations, Cambridge University Press, Cambridge, 2000.

[25] I. Lasiecka and R. Triggiani, Sharp Trace Estimates of Solutions to Kirchhoff and Euler-Bernoulli Equations, Appl. Math Optim 28 (1993), pp. 277-306.

[26] T.F. Ma, Boundary stabilization for a non-linear beam on elastic bearings, Math. Meth. Appl. Sci. 24 (2001), pp. 583-594.

[27] A. Miranville, S. Zelik, Attractors for dissipative partial differential equations in bounded and unbounded domains. In: M.C. Dafermos and M. Pokorny, (eds) Handbook of Differential Equations: Evolutionary Equations, v.4, Elsevier, Amsterdam, 2008.

[28] J.L. Nowinski and H. Ohnabe, On certain inconsistencies in Berger equations for large deflections of plastic plates, Int. J. Mech. Sci 14 (1972), pp. 165-170.

[29] J.-P. Puel and M. Tucsnak, Boundary stabilization for the von Karman equations, SIAM J. Control and Optim. 33 (1995), 1, pp. 255-273.

[30] G. Raugel, Global attractors in partial differential equations, In: Fiedler, B. (ed.) Handbook of Dynamical Systems, v. 2, Elsevier Sciences, Amsterdam, 2002.

[31] R.E. Showalter, Monotone Operators in Banach Spaces and Nonlinear Partial Differential Equations, v. 49, AMS, 1997.

[32] C.P. Vendhan, A study of Berger equations applied to nonlinear vibrations of elastic plates, Int. J. Mech. Sci 17 (1975), pp. 461-468. 